\title{
Functional Micro-Organization of Primary Visual Cortex: Receptive Field Analysis of Nearby Neurons
}

\author{
Gregory C. DeAngelis, Geoffrey M. Ghose, Izumi Ohzawa, and Ralph D. Freeman \\ Vision Science Group, University of California, Berkeley, California 94720-2020
}

It is well established that multiple stimulus dimensions (e.g., orientation and spatial frequency) are mapped onto the surface of striate cortex. However, the detailed organization of neurons within a local region of striate cortex remains unclear. Within a vertical column, do all neurons have the same response selectivities? And if not, how do they most commonly differ and why? To address these questions, we recorded from nearby pairs of simple cells and made detailed spatiotemporal maps of their receptive fields. From these maps, we extracted and analyzed a variety of response metrics. Our results provide new insights into the local organization of striate cortex. First, we show that nearby neurons seldom have very similar receptive fields, when these fields are characterized in space and time. Thus, there may be less redundancy within a column than previously thought. Moreover, we show that correlated discharge in-

Columnar organization is a common feature of cortical architecture (Mountcastle, 1997). Neurons along a path perpendicular to the cortical surface often have similar functional properties, and these properties often vary systematically across the surface of the cortex. In primary visual (or striate) cortex, systems of columns are well documented for orientation preference, ocular dominance, and retinotopic location (Hubel and Wiesel, 1977). Preferred spatial frequency also has an orderly representation, although the details of this organization remain controversial (Maffei and Fiorentini, 1977; Tootell et al., 1981; Berardi et al., 1982; Tolhurst and Thompson, 1982; Bonhoeffer et al., 1995; Shoham et al., 1997). Directionality columns have also been reported (Payne et al., 1980; Tolhurst et al., 1981; Berman et al., 1987; but see Bonhoeffer et al., 1995; Shmuel and Grinvald, 1996; Weliky et al., 1996). It remains unknown, however, whether there are columns for other important response properties of striate neurons, such as receptive field (RF) shape, response latency, and temporal frequency selectivity. Although the extant literature suggests that some response parameters are clustered (usually in the form of columns) and others are not, a quantitative comparison of the degree of clustering has not been done for a wide range of parameters.

\footnotetext{
Received July 1, 1998; revised Jan. 15, 1999; accepted Feb. 16, 1999.

This work was supported by research and CORE grants from the National Eye Institute (EY01175 and EY03176). We thank Akiyuki Anzai, Crista Barberini, Greg Horwitz, Bill Newsome, Jamie Nichols, and Eyal Seidemann for helpful discussions and comments on this manuscript.

Correspondence should be addressed to Dr. Ralph D. Freeman, University of California, 360 Minor Hall, Berkeley, CA 94720-2020.

Dr. DeAngelis' present address: Department of Neurobiology, Stanford University School of Medicine, Stanford, CA 94305-5401.

Dr. Ghose's present address: Division of Neuroscience, Baylor College of Medicine, One Baylor Plaza, Houston, TX 77030.

Copyright (C) 1999 Society for Neuroscience 0270-6474/99/194046-19\$05.00/0
}

creases with receptive field similarity; thus, the local dissimilarity between neurons may allow for noise reduction by response pooling. Second, we show that several response variables are clustered within striate cortex, including some that have not received much attention such as response latency and temporal frequency. We also demonstrate that other parameters are not clustered, including the spatial phase (or symmetry) of the receptive field. Third, we show that spatial phase is the single parameter that accounts for most of the difference between receptive fields of nearby neurons. We consider the implications of this local diversity of spatial phase for population coding and construction of higher-order receptive fields.

Key words: visual cortex; receptive field; neuron; correlation; columnar organization; reverse correlation; phase coding

Columnar organization is most commonly studied using singleunit recordings (e.g., Hubel and Wiesel, 1974), metabolic labeling (e.g., Hubel et al., 1977), and optical imaging techniques (e.g., Ts'o et al., 1990). In most of these studies, the approach is to map how a small number of response variables change across the surface of the cortex. For example, some studies have examined the joint layout of orientation and ocular dominance columns (e.g., Payne and Berman, 1983; Bartfeld and Grinvald, 1992; Obermayer and Blasdel, 1993) or orientation and direction domains (Berman et al., 1987; Shmuel and Grinvald, 1996; Weliky et al., 1996), and a recent study (Hübener et al., 1997) has examined the relationships between three systems of columns (orientation, ocular dominance, and spatial frequency). With these methodologies, however, it has not been possible to characterize more than a few response properties simultaneously. Hence, little is known about the inter-relationships between many different parameters within a column.

Not all aspects of functional organization can be studied by all methods. For example, it is currently not possible to study response parameters such as visual latency and receptive field shape (phase) using imaging techniques. Moreover, although metabolic labeling and imaging techniques can visualize columns in vivo, their resolution is too coarse to say anything about the functional diversity of neurons within a column. In this study, we adopt an approach that allows us to measure many response properties of single neurons and enables us to ask detailed, quantitative questions about functional diversity within cortical columns.

Knowledge of the micro-organization of response properties within a cortical column has important implications for population coding of visual information. In an orientation column, for example, there are thousands of neurons with similar orientation preference and receptive field location. Therefore, nearby neu- 
rons may provide highly redundant signals concerning the visual scene. Alternatively, neurons within an orientation column may vary substantially along other response dimensions (e.g., receptive field shape) that typically are not, or cannot be, measured in studies of functional architecture. Thus, to evaluate the amount of redundancy within the population code, it is necessary to characterize the responses of neurons with a method that captures the full range of the sensitivity of a neuron in a multidimensional parameter space (see also, Gawne and Richmond, 1993).

In this study, we have used an approach that is complementary to that of previous investigations. Instead of describing how a single response variable changes across a large patch of cortex, we have measured a broad array of response properties for pairs of nearby simple cells. This is achieved by measuring complete spatiotemporal RF profiles with the use of a reverse correlation technique (Jones and Palmer, 1987; DeAngelis et al., 1993a). Because simple cells exhibit approximately linear spatiotemporal summation (Movshon et al., 1978; DeAngelis et al., 1993b), these measurements yield accurate estimates of many useful response properties, including metrics such as RF size and shape, response latency, spatial and temporal frequency tuning, and direction selectivity. Note, however, that multi-input techniques are required to extract similar data from complex cells (Gaska et al., 1994); thus, we focus exclusively on simple cells here. From our measurements, we can determine which RF parameters are clustered within a local region of striate cortex. A weakness of our approach, however, is that we cannot distinguish columnar organization from other possible forms, such as laminar organization, because we only record from nearby cells. Nevertheless, this method can unambiguously identify those parameters that are not clustered, and thus not organized in columns.

In this report, we address the following five questions. (1) How similar are the spatiotemporal RFs of nearby neurons? Our results show that RFs of nearby simple cells are seldom very similar when compared in the space-time domain, suggesting that there may be less redundancy in the population code than previously thought. (2) Which RF parameters are clustered within a cortical column? The strength of clustering varies widely among the different response parameters. Orientation and spatial frequency show the strongest clustering, and there is modest clustering for preferred temporal frequency, response latency, and response duration. We find no evidence of clustering, however, for the spatial phase of the RF. (3) When nearby RFs differ, which parameters account for most of the difference? The single most important factor distinguishing RFs of nearby cells is spatial phase. Most other RF parameters account for little cell to cell variation within a local region of striate cortex. (4) How do the above aspects of functional organization change during postnatal development? Our results show that the micro-organization of striate cortex is quite mature at postnatal age 4 weeks. (5) What are the functional implications of local diversity in spatial phase? We suggest that this diversity may permit efficient construction of complex cell RFs from simple cell inputs. In addition, our finding that neurons with similar RFs tend to have correlated responses suggests that pooling across nearby simple cells with different spatial phases may yield an improvement in signal-to-noise ratio.

\section{MATERIALS AND METHODS}

Procedures for animal preparation and maintenance, surgery, single-unit recording, and RF mapping have been described in detail elsewhere (DeAngelis et al., 1993a, 1995a). Only a brief account is provided here, with an emphasis on those aspects of the methodology most relevant to the present study. All animal care and experimental guidelines conformed to those established by the National Institutes of Health.

Experiments were performed with adult cats and kittens at 4 postnatal weeks. Under halothane (1.5-3\% in $\mathrm{O}_{2}$ ) anesthesia, a tracheostomy was performed, and a tracheal tube was inserted. The animal was then placed in a stereotaxic frame and secured with ear bars and a mouth bar. For adult cats, a $5 \mathrm{~mm}$ craniotomy was centered at Horsley-Clarke coordinates P4 L2, and the dura was reflected. For 4-week-old kittens, the craniotomy was centered $2-3 \mathrm{~mm}$ anterior and $1-2 \mathrm{~mm}$ lateral to the branch point of the lambda suture. Paralysis was induced with a loading dose of gallamine triethiodide (Flaxedil) and maintained with a continuous infusion $\left(10 \mathrm{mg} \cdot \mathrm{kg}^{-1} \cdot \mathrm{hr}^{-1}\right)$ of Flaxedil. Sodium thiamylal (Surital) was also infused at a rate of $1 \mathrm{mg} \cdot \mathrm{kg}^{-1} \cdot \mathrm{hr}^{-1}$ to maintain an adequate level of anesthesia. Artificial respiration was performed with a gas mixture of $70 \% \mathrm{~N}_{2} \mathrm{O}, 29 \% \mathrm{O}_{2}$, and $1 \% \mathrm{CO}_{2}$. Vital signs (ECG, EEG, and expired $\mathrm{CO}_{2}$ level) were recorded continuously (at 5 min intervals) by a PC-based physiological monitoring system (Ghose et al., 1995).

To record the activity of single units from striate cortex (area 17), tungsten-in-glass electrodes were lowered into a region of cortex exposed by craniotomy. Electrode penetrations were made at oblique angles to the cortical surface and usually traversed $4-5 \mathrm{~mm}$ along the medial bank of the postlateral gyrus. Agar at $38^{\circ} \mathrm{C}$ was applied around the electrodes to prevent desiccation, and melted wax was layered over the agar to create a sealed chamber and reduce cortical pulsation. In early experiments, spike sorting was achieved using an amplitude-based window discriminator with two reference levels (allowing two single units with different spike amplitudes to be discriminated), and spike occurrences were recorded with $1 \mathrm{msec}$ resolution. In later experiments, spike times were recorded with $40 \mu \mathrm{sec}$ resolution, and spikes were sorted using a custommade device (Ohzawa et al., 1996). This custom spike sorter allows the experimenter to isolate as many as five single units from a single electrode by defining a series of voltage-time constraint points. This latter method allowed us to easily discriminate spike waveforms on the basis of shape, as well as amplitude. All neuronal pairs discussed in this paper were recorded from the same electrode.

Visual stimuli were generated by computer and displayed on a pair of video monitors, one for each eye, that the cat viewed by means of beam splitters. The video displays (Mitsubishi Electronics) had a resolution of $1024 \times 804$ pixels, subtending $28^{\circ} \times 22^{\circ}$ at a viewing distance of $57 \mathrm{~cm}$, and were refreshed at $76 \mathrm{~Hz}$. The mean luminance of the displays, as viewed through the partially reflecting beam splitters, was $12 \mathrm{~cd} / \mathrm{m}^{2}$.

Experiments typically lasted for $4 \mathrm{~d}$. At the end of an experiment, the animal was administered an overdose of pentobarbital sodium (Nembutal), and cortical tissue was prepared for histological examination. Electrode tracks were reconstructed, and cortical laminae were identified. This analysis confirmed that all cells were recorded from area 17 and that the majority of simple cells were recorded from layers 3,4 , and 6 .

Experimental protocol. When we were able to simultaneously isolate action potentials from two or more simple cells, we performed the following battery of tests. First, the preferred orientation and spatial frequency of each cell, as well as the size and location of its RF, were estimated using a computerized "search" program. Next, quantitative measurements of the orientation and spatial frequency tuning of each cell were obtained by presenting randomized sequences of drifting sinusoidal gratings in which one of these parameters was systematically varied. Each grating was presented for a period of $4 \mathrm{sec}$, during which peristimulus time histograms of the responses were accumulated. Stimuli of each orientation or spatial frequency were presented 4-6 times, and responses were averaged. For binocular cells, tuning curves were measured for each eye by randomly interleaving left and right eye stimuli.

To measure spatiotemporal RF profiles for pairs of simultaneously recorded simple cells, we used a reverse correlation technique (Jones and Palmer, 1987). In this method, the visual stimulus is a sequence of small bright and dark rectangular bars that are flashed in rapid succession at randomly chosen locations on a two-dimensional stimulus grid. The stimulus grid, which typically consisted of $20 \times 20$ spatial locations, was centered over the RFs of the recorded neurons and was large enough to cover the entire RFs. Both the stimulus grid and the small bar stimuli were oriented to match the preferred orientation of the cells, as measured with drifting gratings. When the preferred orientations of a pair of cells differed somewhat (see Fig. $11 \mathrm{~A}$ ), we chose a stimulus orientation that roughly split the difference. The bar stimuli were typically $\sim 1.5^{\circ}$ long (range, $1.0-2.0^{\circ}$ ) and $0.5^{\circ}$ wide (range, $0.3-1.2^{\circ}$ ) and were usually presented for a duration of $40 \mathrm{msec}$ (range, $26-65 \mathrm{msec}$ ). However, these 


\section{Cell 1}

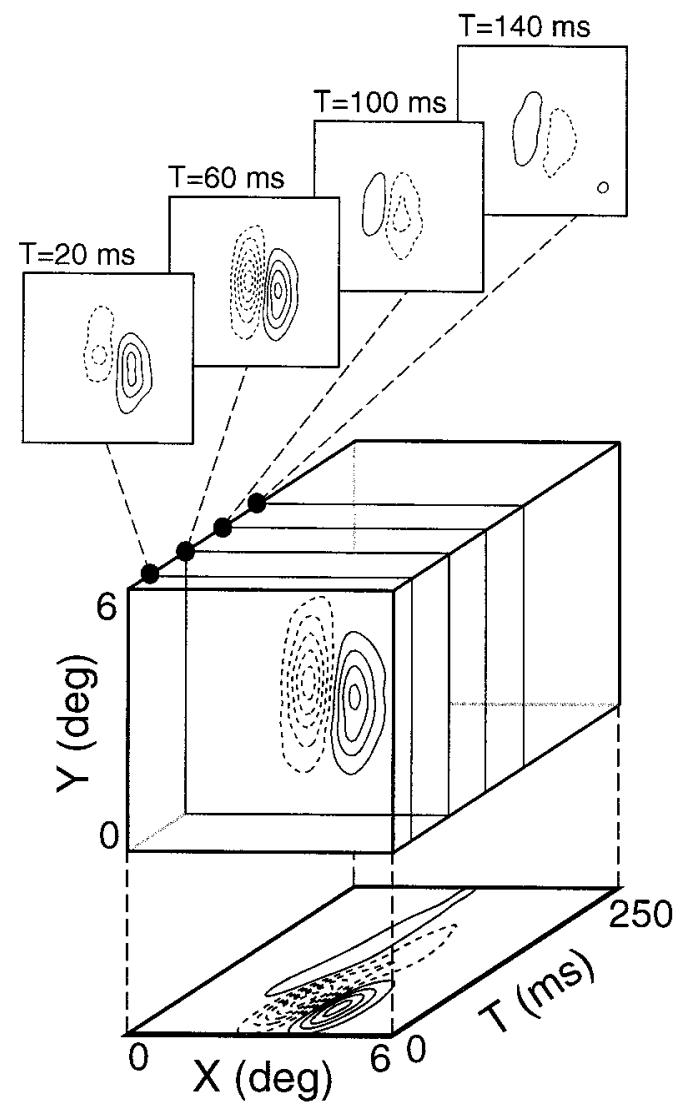

\section{Cell 2}

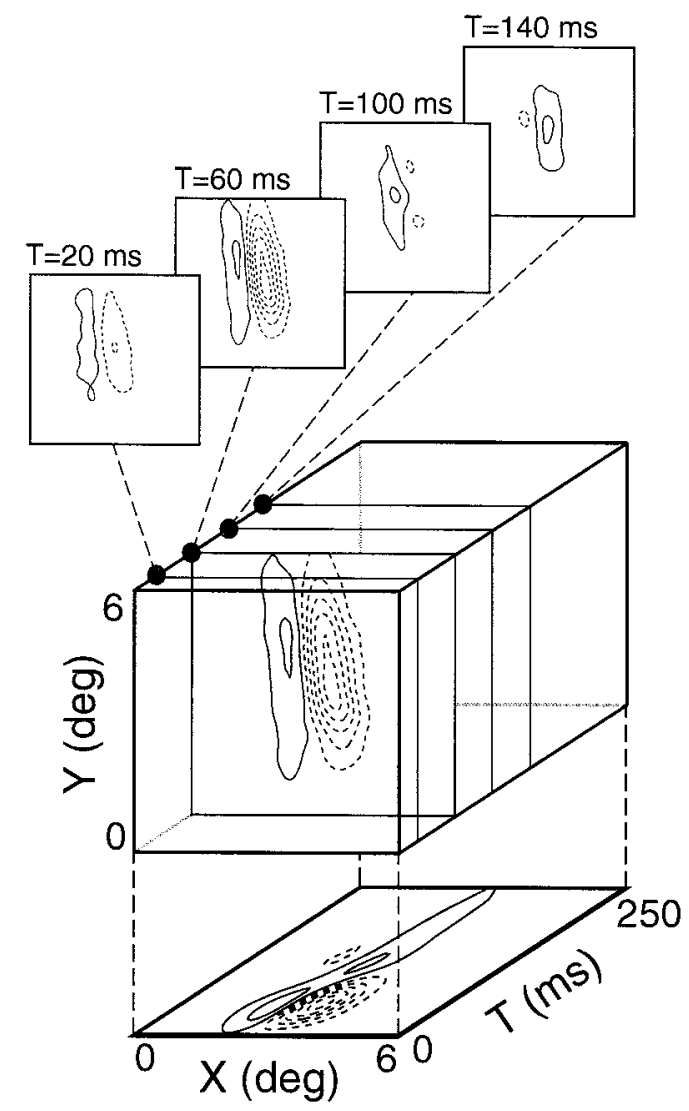

Figure 1. Spatiotemporal RF profiles for a pair of simple cells recorded simultaneously from the same microelectrode. Each RF profile describes the sensitivity of the cell to luminance increments and decrements as a function of space $(X, Y)$ and time $(T)$. For each neuron, four spatial $(X-Y)$ cross sections, taken at equally spaced time increments, are shown (top). In addition, each RF profile is summarized as an $X$-T plot (bottom) by integrating the $X-Y-T$ data along the $Y$-axis, which is approximately parallel to the preferred orientation of each cell. Each $X-Y$ or $X-T$ profile is plotted as an isoamplitude contour map, in which solid contours represent responses to luminance increments, and dashed contours denote responses to luminance decrements. For additional details concerning the construction of RF profiles, see our previous papers (DeAngelis et al., 1993a, 1995a; Ohzawa et al., 1996). For this pair of simple cells, the $X-Y-T$ SI is 0.17 (see Results for details).

parameters were adjusted according to the spatial and temporal resolution of the responses of the cells, to elicit the largest responses possible without causing any substantial blurring of the RF profiles (DeAngelis et al., 1993a). When a pair of binocular simple cells was recorded, we generally mapped RFs by stimulating through the eye that yielded the largest responses. In a few cases, we also mapped RFs through the other eye (see Fig. 17).

To construct spatiotemporal RF maps, such as those shown in Figure 1 , we compute a cross-correlation between the stimulus sequence and the recorded spike trains over a range of correlation delays between stimulus and response (see DeAngelis et al., 1993a; McLean et al., 1994; Ohzawa et al., 1996, for additional details). If there is coupling between the stimulus and response at a particular correlation delay $(\mathrm{T})$, then a spatial pattern of bright- and dark-responsive subregions will emerge in the RF profiles (Fig. 1, top); otherwise, the profiles will show no structure. Details of the theory and assumptions behind this technique are discussed elsewhere (DeBoer and Kuyper, 1968; Jones and Palmer, 1987; DeAngelis et al., 1993a). For the present purpose, we note that the RF profile obtained from a simple cell using this method is roughly equivalent to the spatiotemporal impulse response of the cell (DeAngelis et al., 1995b; Ohzawa et al., 1996). Moreover, because simple cells behave linearly under steady-state conditions, these RF profiles can be used to predict, with reasonable accuracy, the responses of simple cells to a variety of different stimuli (DeAngelis et al., 1993b; McLean et al., 1994).

In this study, we sometimes mapped RFs using a spatially onedimensional (1-D) variant of the reverse correlation stimulus. The visual stimuli in these cases were long, thin bars (slightly longer than the RF in length, $0.3-1.0^{\circ}$ in width) that were presented at 20 different positions along the axis perpendicular to the preferred orientation of the cells. Thus, the RF profile obtained in these cases is a function of only one dimension of space, instead of two (i.e., the raw data form an $X-T$ plot, instead of an $X-Y-T$ plot; see Fig. 1). This 1-D version of the method was often used to map RFs of neurons from 4-week-old kittens [for which response rates are generally much lower than adults (Freeman and Ohzawa, 1992)], because the long bar stimuli elicit a better response than short bars. We also used the 1-D version of the stimulus to reduce the overall recording time when it was difficult to maintain adequate isolation of two action potentials for a prolonged period of time.

\section{RESULTS}

Altogether, we obtained complete data sets for 132 cells. A complete data set consisted of an $X-Y-T$ or $X-T$ profile and, in all but 19 cases, responses to sine-wave gratings of variable orientation and spatial frequency. This total population consisted of 39 pairs of simple cells and two triplets (for a total of 45 pairings) from 21 adult cats, along with 21 pairs of simple cells recorded from 10 kittens at 4 postnatal weeks. The number of cell pairs recorded from each animal was small because many single neurons from these animals were recorded for other studies. Repre- 
sentative data are shown in Figure 1 for a pair of simple cells whose spike trains were recorded simultaneously in response to a pseudorandom reverse-correlation stimulus (see Materials and Methods). The data are presented in an $X-Y-T$ coordinate system, depicting two dimensions of space and one of time. Note that $X$ and $Y$ are always defined such that the $Y$-axis is parallel to the preferred orientation of the neuron, and the $X$-axis is orthogonal. Spatial $(X-Y)$ RF profiles are shown for four different time delays (top). Contour density depicts response strength, and it is clear in this case that the strongest response was obtained for a correlation delay of $60 \mathrm{msec}$. If we integrate along the axis parallel to that of the preferred orientation of the cell (the $Y$-axis of the cube), we are left with an $X-T$ representation (bottom). For further details about these spatiotemporal profiles, see DeAngelis et al. (1993a) and Ohzawa et al. (1996).

The major question that we address in this study is: how similar are the spatiotemporal RFs of nearby pairs of simple cells, and in which respects do they differ? For the cell pair of Figure 1, it is clear that the two RF profiles have a similar time course and that both cells exhibit a moderate degree of space-time inseparability in their $X-T$ profiles [an $X-T$ profile, $\mathrm{R}(X, T)$, that is space-time inseparable cannot be described as the product of a spatial profile, $\mathrm{G}(X)$, and a temporal profile, $\mathrm{H}(T)[\mathrm{R}(X, T) \neq \mathrm{G}(X) \times \mathrm{H}(T)]$. As shown previously, most simple cells in striate cortex do not have space-time separable RFs. Neurons with inseparable RFs tend to be direction-selective, whereas those with separable RFs generally do not (McLean and Palmer, 1989; DeAngelis et al., 1993a; McLean et al., 1994)]. This inseparability manifests itself in the form of RF subregions that are tilted to the right in the $X-T$ domain (seen most clearly in Fig. $2 A$, which shows $X-T$ data for the same pair of cells). These two cells differ mainly in the spatial structure (or symmetry) of their RFs. This difference can be observed in each of the four spatial profiles shown at the top of Figure 1. For example, at $T=60 \mathrm{msec}$, cell 1 has a dark-excitatory RF subregion (dashed contours) to the left of a bright-excitatory region (solid contours), whereas cell 2 has a weak bright-excitatory region to the left of a stronger dark-excitatory region.

Although differences in RF structure between the two neurons of Figure 1 are clear by inspection, we want to quantify and summarize these differences for a population of cell pairs. In what follows, we describe two methods of analysis that provide complementary information. The first method is model-free and provides an estimate of the degree of similarity between two RF profiles. This method has the advantage of simplicity, but it cannot tell us how two RFs are different. The second method involves fitting a model to the space-time RF profiles of pairs of neurons. Although more complicated, this analysis allows us to make quantitative, parametric comparisons between pairs of cells.

\section{Model-free analysis of similarity}

To quantify the degree of similarity between RFs of two neurons, we compute a similarity index (SI) as follows:

$$
\mathrm{SI}_{S}=\frac{\sum_{S}(U(S) * V(S))}{\sqrt{\sum_{S} U(S)^{2} \sum_{S} V(S)^{2}}},
$$

where $U(S)$ and $V(S)$ are RF profiles for a pair of neurons, and $S$ is the N-dimensional space in which the receptive fields are defined. The numerator of this quantity is simply the inner
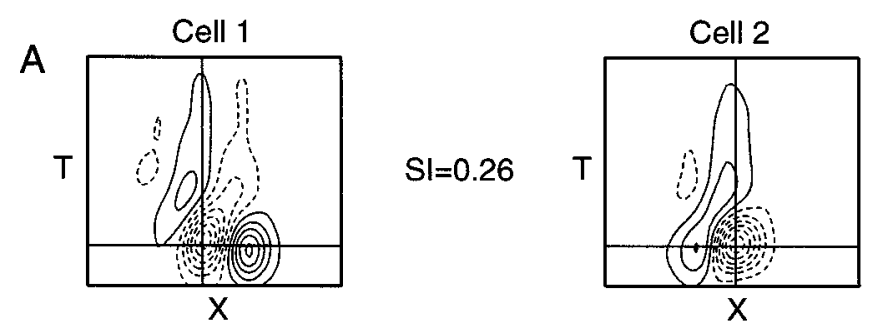

B
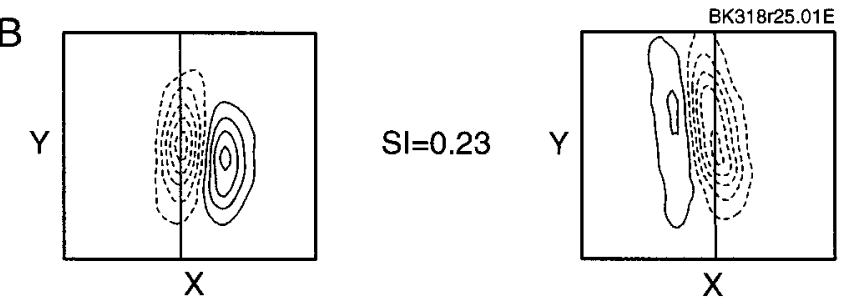

C

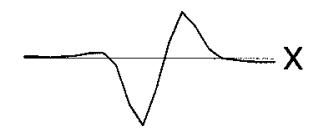

$\mathrm{SI}=0.33$

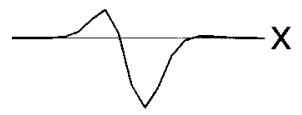

D

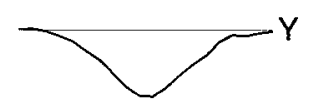

$\mathrm{SI}=0.96$

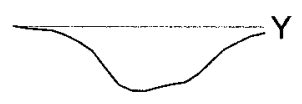

$E$
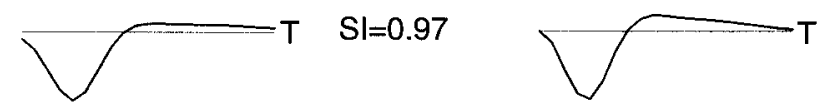

Figure 2. Receptive field cross sections and their corresponding SIs are shown for the same pair of simple cells depicted in Figure 1. $A, X-T$ profiles. The similarity index computed in the $X-T$ plane is $\mathrm{SI}_{X-T}=0.26$. $B, X-Y$ cross sections taken at $T=60 \mathrm{msec}$. $\mathrm{SI}_{X-Y}=0.23$. $C$, Onedimensional spatial cross sections taken parallel to the $X$-axis (i.e., perpendicular to the preferred orientation axis of the cells). Each $X$ cross section was taken at $T=60 \mathrm{msec}$, as shown by the horizontal lines through the $X-T$ profiles in $A$. The $\mathrm{SI}_{X}$ is 0.33 , reflecting the fact that there is a clear difference in spatial phase between $X$ profiles for the two cells. $D$, One-dimensional spatial cross sections taken parallel to the $Y$-axis. The $X$-coordinate for each $Y$ cross section is shown by the vertical lines through the $X-Y$ profiles in $B ; T=60 \mathrm{msec} . \mathrm{SI}_{Y}$ is 0.96 , indicating that the $Y$ cross sections for the two cells are quite similar. $E$, Temporal cross sections are shown. Vertical lines through the $X-T$ data in $A$ give the $X$ values at which these cross sections were obtained. Note that the two temporal profiles have a similar shape, resulting in an $\mathrm{SI}_{T}$ value of 0.97 .

product of the two RF profiles, and the denominator normalizes the index to the range from -1.0 to 1.0. If the two RF profiles are identical but one is the inverse of the other, then $\mathrm{SI}_{\mathrm{S}}=-1.0$. If $U(S)$ and $V(S)$ are identical, $\mathrm{SI}_{\mathrm{S}}=1.0$. If, for example, $U(S)$ and $V(S)$ form a quadrature pair (i.e., they differ in phase by $90^{\circ}$ ), then $\mathrm{SI}_{\mathrm{S}}=0$. For the example of Figure 1, where the two RF profiles clearly differ in spatial phase, $\mathrm{SI}_{X-Y-T}=0.17$.

By computing similarity indices for various cross sections through the $X-Y-T$ data, we can compare the degree to which two RFs differ along various dimensions. Figure 2 shows five different cross sections through the data of Figure 1. Panels $A$ and $B$ show two-dimensional (2-D) cross sections ( $X-T$ and $X-Y$, respectively) that are obtained by slicing through the $X-Y-T$ data at the optimal values of $Y$ and $T$, respectively. Panels $C-E$ show one-dimensional cross sections through the overall peak in the $X-Y-T$ data. The $X$ and $T$ cross sections (Fig. $2 C, E$ ) are obtained by slicing through the peak of the $X-T$ data (horizontal and vertical lines, respectively, through the profiles in Fig. $2 A$ ). Similarly, the $Y$ cross section is 
A
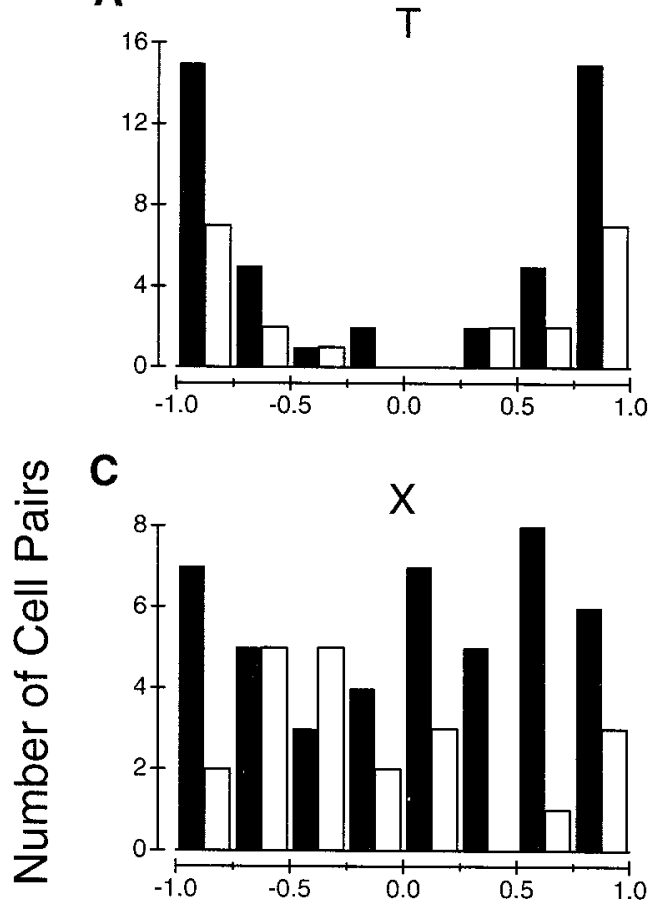

E

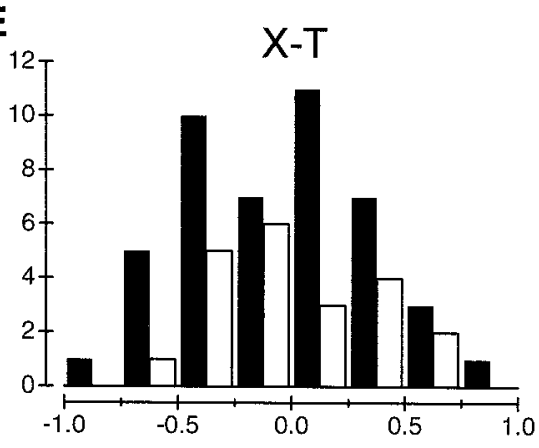

B

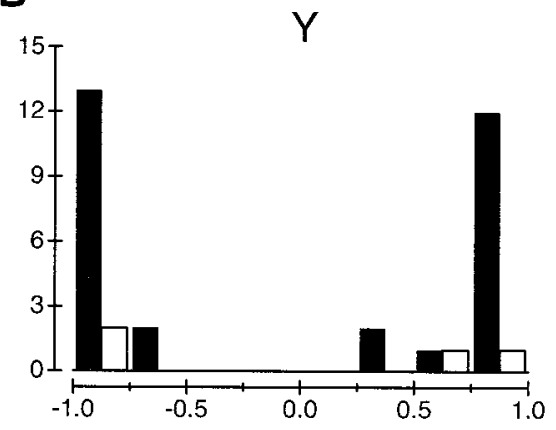

D

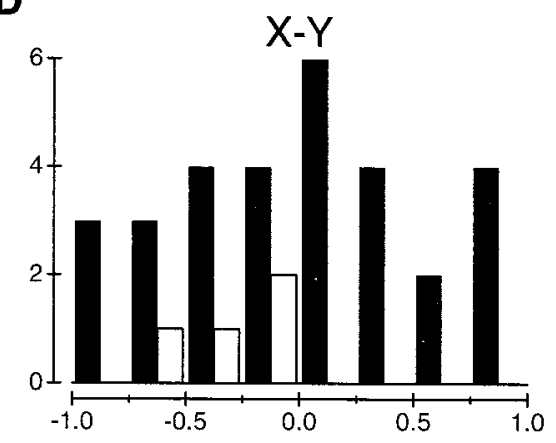

F

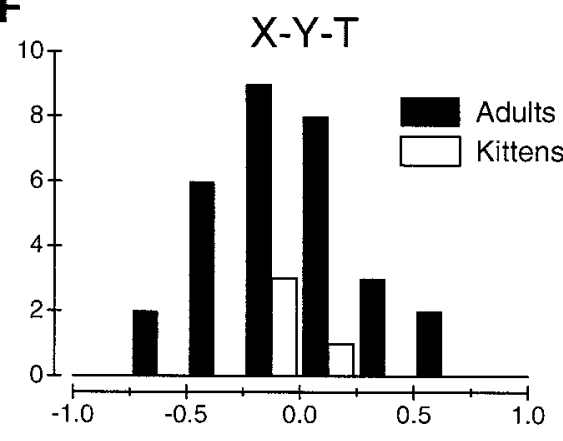

Similarity Index

obtained by slicing through the peak of the $X-Y$ data (Fig. $2 B$, vertical line).

Comparison of similarity indices for the five cross sections of Figure 2 reveals that most of the difference between this pair of RFs occurs along the $X$ dimension. The $Y$ and $T$ cross sections for the two cells are quite similar $\left(\mathrm{SI}_{Y}=0.96\right.$ and $\left.\mathrm{SI}_{T}=0.97\right)$, whereas the $X$ cross sections have markedly different shapes $\left(\mathrm{SI}_{X}\right.$ $=0.33$ ). Moreover, the 2-D cross sections have only moderately lower similarity indices than the $X$ cross section $\left(\mathrm{SI}_{X-T}=0.26\right.$, $\mathrm{SI}_{X-Y}=0.23$ ). This pattern of results is typical of most of the pairs of simple cells that we recorded from both adult cats and kittens.

Before examining population data, it is important to point out that our 1-D and 2-D cross sections were not chosen to maximize or minimize the similarity index. It is clear, for example, that the shape of the $X$ cross section will generally depend on the values of $T$ and $Y$ at which the cross section is taken. To avoid subjective bias, we always took cross sections through the location of the absolute maximum in the $X-Y-T$ data (or through the overall maximum in the $X-T$ data when the mapping stimuli were one- dimensional; see Materials and Methods). Thus, by adopting a consistent criterion, we are able to pool data across cell pairs.

Figure 3 shows distributions of similarity indices for all pairs of neurons in our sample. Despite the fact that 1-D cross sections were not chosen to maximize SI, the distributions of $\mathrm{SI}_{T}$ and $\mathrm{SI}_{Y}$ (Fig. 3A,B, respectively) are strongly bimodal, with most of the values near 1.0 or -1.0 . Thus, $T$ and $Y$ cross sections tend to be nearly identical in shape for simultaneously recorded pairs of simple cells (allowing for an inversion of sign, which occurs when the strongest subregions of the two RFs have opposite polarities; e.g., see Fig. 14A). In contrast, the distribution of $\mathrm{SI}_{X}$ (Fig. $3 C$ ) is roughly uniform, showing that many pairs of nearby simple cells differ substantially along the $X$ dimension. Distributions of $\mathrm{SI}_{X-Y}$ (Fig. $3 D$ ) and $\mathrm{SI}_{X-T}$ (Fig. $3 E$ ) also include a broad range of values, but are shifted somewhat more toward an SI of zero. Finally, the distribution for the full $X-Y-T$ data (Fig. $3 F$ ) is centered around an $\mathrm{SI}_{X-Y-T}$ of zero. Note, in particular, the lack of data points near 1.0 and -1.0 in the $\mathrm{SI}_{X-Y-T}$ distribution, indicating that the RFs of nearby simple cells are generally not very similar when one considers the full $X-Y-T$ profiles. Although the sample of cell 


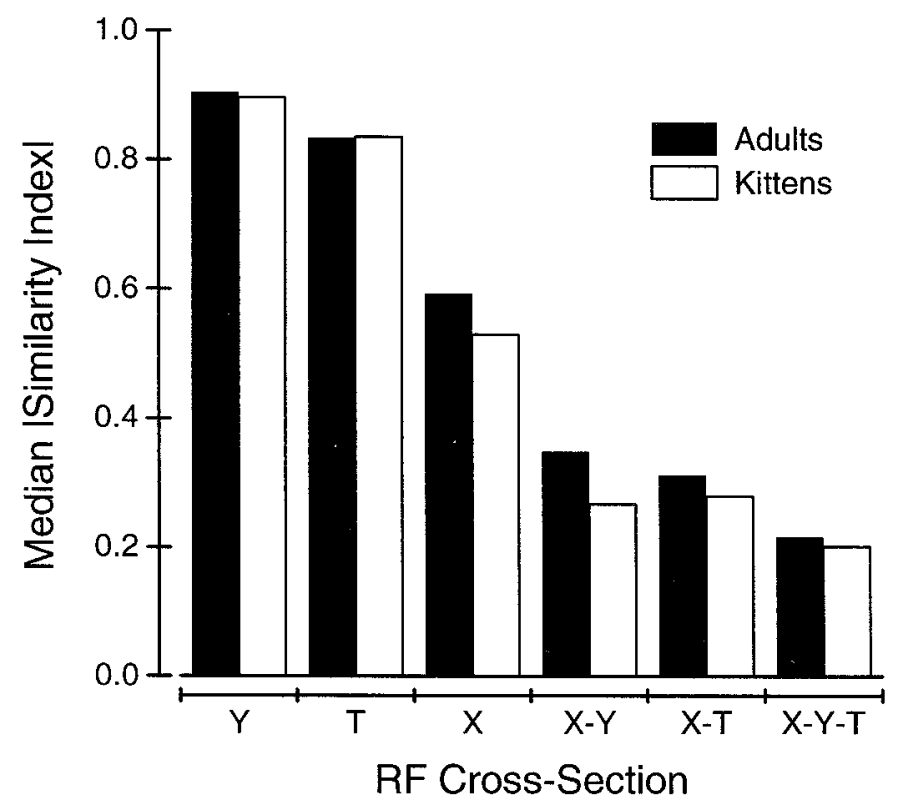

Figure 4. Quantitative summary of RF similarity indices as a function of the dimensionality of the RF cross section. The height of each bar gives the median absolute value of SI for the populations of cell pairs in Figure 3 (i.e., each histogram in Fig. 3 is folded about the SI $=0$ axis, and the median value of the resultant distribution is computed). Open and filled bars denote population data obtained from kittens and adult cats, respectively.

pairs from 4-week-old kittens is substantially smaller than that for adults, the data of Figure 3 show the same basic pattern for the two age groups.

Figure 4 summarizes the SI data. In this graph, the median absolute value of the SI is plotted for each different RF cross section (sorted in decreasing order). Two-factor ANOVA reveals a highly significant main effect of RF cross section $(F=54.8 ; p \ll$ $0.001)$, with no significant difference between cats and kittens $(F=1.89 ; p=0.17)$. The median $\mathrm{SI}_{X}$ is significantly smaller than the median values of $\mathrm{SI}_{Y}$ and $\mathrm{SI}_{T}$ (Mann-Whitney $U$ test; $p \ll$ 0.001 for both comparisons, cats and kittens pooled), and the median $\mathrm{SI}_{X}$ is significantly larger than the median values for $\mathrm{SI}_{X-Y}$ $(p=0.012), \operatorname{SI} X-T(p<0.001)$, and $\mathrm{SI}_{X-Y-T}(p<0.001)$. For the full $X-Y-T$ data, the median SI values are 0.22 and 0.20 for cats and kittens, respectively. The pooled median value of $\mathrm{SI}_{X-Y-T}$ is significantly smaller $(p<0.01)$ than that for all other cross sections, except the $X-T$ cross section $(p=0.094)$.

\section{Receptive field similarity and correlated discharge}

Thus far, we have examined the RF of each simple cell separately and have shown that nearby pairs of neurons are often dissimilar. Here, we examine whether there is a relationship between RF similarity and the joint firing of two nearby neurons. The spike discharges of cell pairs with similar RFs might be correlated because they share sources of common input. For example, if two simple cells each have an ON (bright) subregion at a given position in visual space, then both cells may be expected to receive input from LGN neurons with an ON-center at that point in space (Reid and Alonso, 1995). Alternatively, responses of a pair might be correlated if one neuron provides direct input to the other. In both of these cases, we would expect correlated discharge to be strongest between pairs of neurons with similar RFs.

To examine the relationship between RF similarity and corre- lated discharge, we constructed cross-correlograms from the responses of each pair of neurons to the same visual stimulus used to map their RFs. Figure 5 shows RF profiles and crosscorrelograms for two pairs of simple cells. Figure $5 A$ shows $X-T$ profiles for a pair of cells with fairly similar RFs $\left(\mathrm{SI}_{X-T}=0.70\right)$. The cross-correlogram for this pair (Fig. $5 B$ ) has a sharp peak centered $\sim 8 \mathrm{msec}$ to the left of zero, suggesting polysynaptic excitation from cell 1 to cell 2 . In contrast, Figure $5 D$ shows a correlogram with a broad peak centered at zero, consistent with the interpretation that these two neurons receive common input. The RF profiles associated with this broad correlogram exhibit a substantial degree of similarity (Fig. $5 C ; \mathrm{SI}_{X-T}=0.56$ ).

Note that Figure 5, $B$ and $D$, shows raw correlograms. For eight pairs of neurons, our reverse correlation procedure included repeat presentations of the same visual stimulus pattern, allowing us to construct a shuffled correlogram (i.e., shift-predictor). All but one of these shuffled correlograms was flat, suggesting that peaks observed in the raw correlogram generally have a neural origin. For many pairs, however, we could not construct shift predictors. Thus, in general, we cannot rule out the possibility that some of the correlation is caused by stimulus coordination. Whether the correlation results from neural connectivity or stimulus coordination, two neurons with highly correlated discharges still carry redundant information. Thus, for our purposes (see Discussion), this distinction is not crucial.

Approximately half of the pairs with synchronous discharges exhibited sharp peaks with latencies and widths on the order of a few milliseconds. Since we have never observed similar features in shuffled correlograms, these sharp peaks appear to indicate monosynaptic connections (Ghose et al., 1994a). Because sharp peaks were only seen between cells with quite similar $\mathrm{RFs}\left(\mathrm{SI}_{X}>\right.$ $0.5)$, these data suggest that excitatory local connections are largely limited to cells with similar functional properties. Thus, local connections, like long-range connections (Ts'o et al., 1986), appear to be functionally specific.

To quantify the strength of correlated discharge for each pair of neurons, we normalized each bin in the raw cross-correlogram by the number of coincidences that one would expect if the two spike trains were independent Poisson processes having the observed firing rates (Melssen and Epping, 1987). We then computed an average over a 5 or $10 \mathrm{msec}$ window centered around the peak in the correlogram. We refer to this quantity as the normalized cross-correlation.

Figure 6 shows that normalized cross-correlation is correlated with RF similarity. SI values for $X, X--T$, and $X-Y-T$ profiles, respectively, are plotted in Figure $6, A-C$. The dependence of normalized cross-correlation on SI is statistically significant (ANCOVA, $F=35.2 ; p<0.0001$ ), and there is no significant interaction between age and similarity index $(F=1.2 ; p=0.27)$, indicating that the slope of the relationship between normalized cross-correlation and SI does not differ between cats (filled circles) and kittens (open circles). Thus, our data show that neurons with similar RFs tend to exhibit more correlated discharge. Of course, one might not expect any correlated discharge between pairs of neurons with opposite RFs (i.e., SI $=-1.0$ ) because such a pair would generally not have temporally overlapping patterns of discharge to a single visual stimulus. However, many pairs of neurons in our sample (including most of those with $-0.5<\mathrm{SI}_{X}$ $<0.5$ in Fig. $6 A$ ) do exhibit temporally overlapping responses to the reverse correlation stimulus, and yet there is little correlation between the discharges. Thus, the lack of strong correlations 


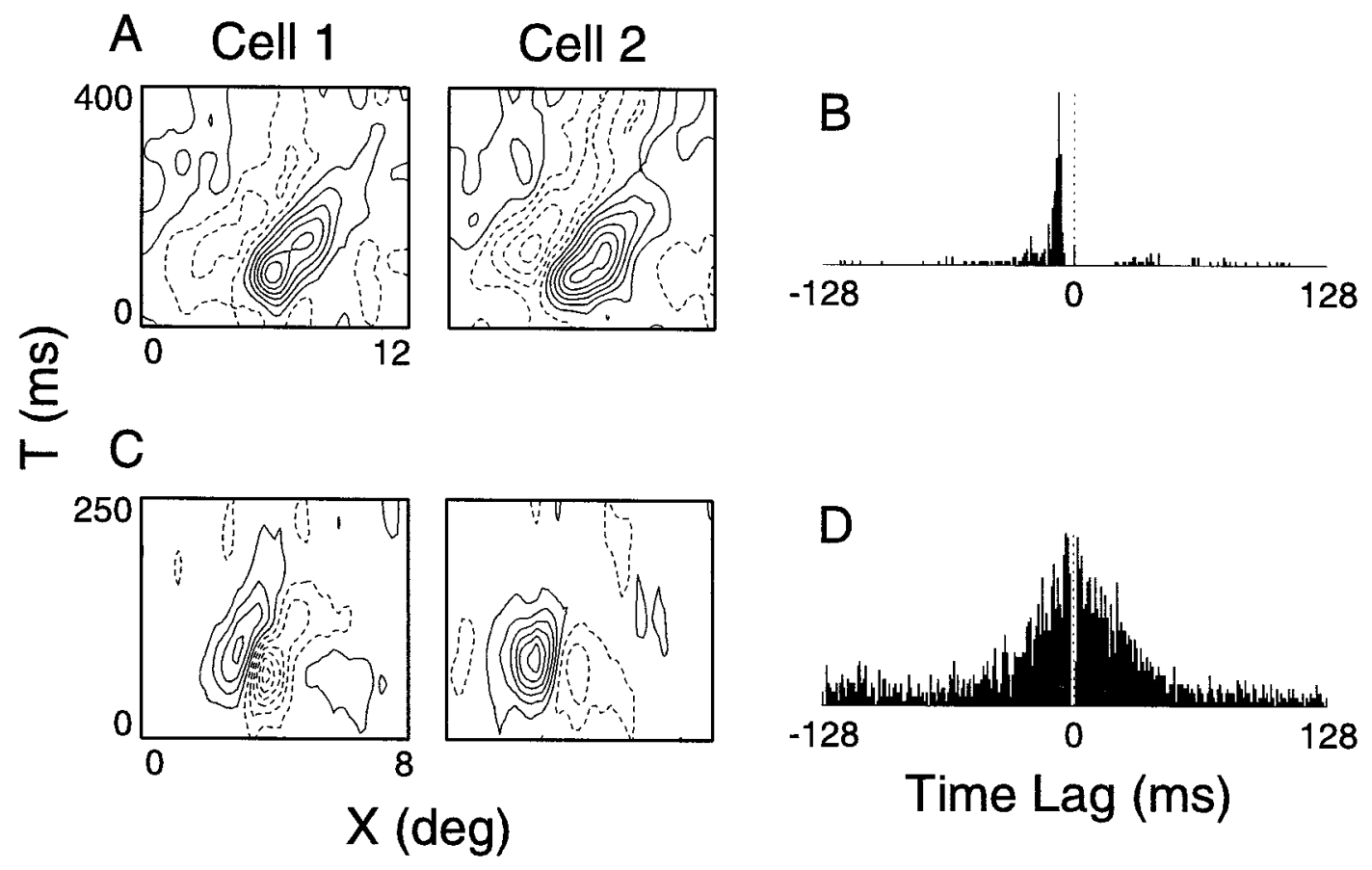

Figure 5. Spatiotemporal RF profiles and cross-correlograms for two pairs of simple cells. $A, X-T$ profiles for a pair of simple cells from a 4-week-old kitten that have similar RFs. $B$, Raw cross-correlogram for the same pair of cells as in $A$. The correlogram was constructed from spike trains recorded during the reverse-correlation mapping experiment. $C, X-T$ profiles for a pair of simple cells recorded from an adult cat. $D$, Raw correlogram for the pair of neurons in $C$. Note that the small notch just to the left of zero is an artifact caused by the fact that the two neurons were recorded simultaneously from a single microelectrode.

between pairs of neurons with dissimilar RFs is not simply caused by a lack of temporal overlap.

The result of Figure 6 is consistent with previous results from our laboratory that showed that the "bicellular" RF, which is an RF map of the correlated spikes between two neurons, can be predicted by the overlap between the RFs of the individual neurons (Ghose et al., 1994b). Given the small average overlap between cells in our sample, correlated activity may potentially provide higher resolution information than is available from the discharge of single neurons. Further implications of these data for population coding are considered in the Discussion.

\section{Parametric (model-based) analysis}

Returning to the SI data, Figures 3 and 4 show clearly that much of the difference between RFs of nearby simple cells can be attributed to variations in RF structure along the $X$ dimension, whereas nearby RFs tend to be quite similar along the $Y$ and $T$ dimensions. Although this analysis provides a useful index of the degree of similarity between two RFs, it does not reveal how two RFs differ. To illustrate this point, Figure 7 shows $X-T$ profiles for two pairs of cells from adult cats; each pair has an $\mathrm{SI}_{X-T}$ value close to zero. It is clear from inspection of the profiles, however, that the two pairs of neurons differ in distinct ways. For the cell pair of Figure $7 A\left(\mathrm{SI}_{X-T}=-0.11\right)$, the two RFs differ in spatial phase by $\sim 90^{\circ}$, but are otherwise quite similar. In contrast, the two cells in Figure $7 B$ clearly prefer opposite directions of motion, as evidenced by the difference in the space-time orientation of the RF subregions (McLean and Palmer, 1989; DeAngelis et al., 1993b). Thus, although these two RFs are similar in other respects, the similarity index is low $\left(\mathrm{SI}_{X-T}=0.13\right)$. We now consider a parametric analysis that allows us to determine how two RFs differ.
We want to characterize the differences between a pair of RFs in terms of physiologically relevant parameters, such as spatial frequency, direction selectivity, latency, etc. Thus, we need to extract a meaningful set of parameters from each spatiotemporal RF profile. We have chosen to accomplish this by developing a RF model that can be fit to the $X-T$ data from simple cells. As described below, this model has a relatively simple architecture, provides excellent fits to the $X--T$ profiles of simple cells, and yields physiologically meaningful parameters.

Figure 8 illustrates the basic structure of the model. An inseparable $\mathrm{RF}, \mathrm{R}(X, T)$ (bottom), is constructed as the weighted sum of two space-time separable components (top), each of which is modeled as the product of a spatial waveform, $\mathrm{G}(X)$, and a temporal waveform, $\mathrm{H}(T)$ :

$$
\left.R(X, T)=K G_{1}(X) H_{1}(T)+\alpha G_{2}(X) H_{2}(T)\right] .
$$

In this formulation, $K$ is an overall scaling factor, and $\alpha$ is a variable weight on the second separable subunit $(-1<\alpha<1)$. When $\alpha=0$, the model RF will be separable; as $\alpha$ approaches 1 , the RF becomes more strongly inseparable [in this formulation, $\alpha$ can be thought of as the linear component of direction selectivity (Albrecht and Geisler, 1991; Reid et al., 1991; DeAngelis et al., 1993a; McLean et al., 1994). In fact, there is a very strong correlation $(r=0.88$; slope $=1.01 ; p<0.001 ; n=132)$ between $\alpha$ and the linear component of direction selectivity, as derived from the spatiotemporal amplitude spectrum (DeAngelis et al., $1993 b)$ of the $X-T$ data]. Negative and positive values of $\alpha$ correspond to opposite preferred directions of motion; thus we refer to $\alpha$ as a direction selectivity index.

The spatial profile, $\mathrm{G}(X)$, of each separable component is modeled as a Gabor function: 


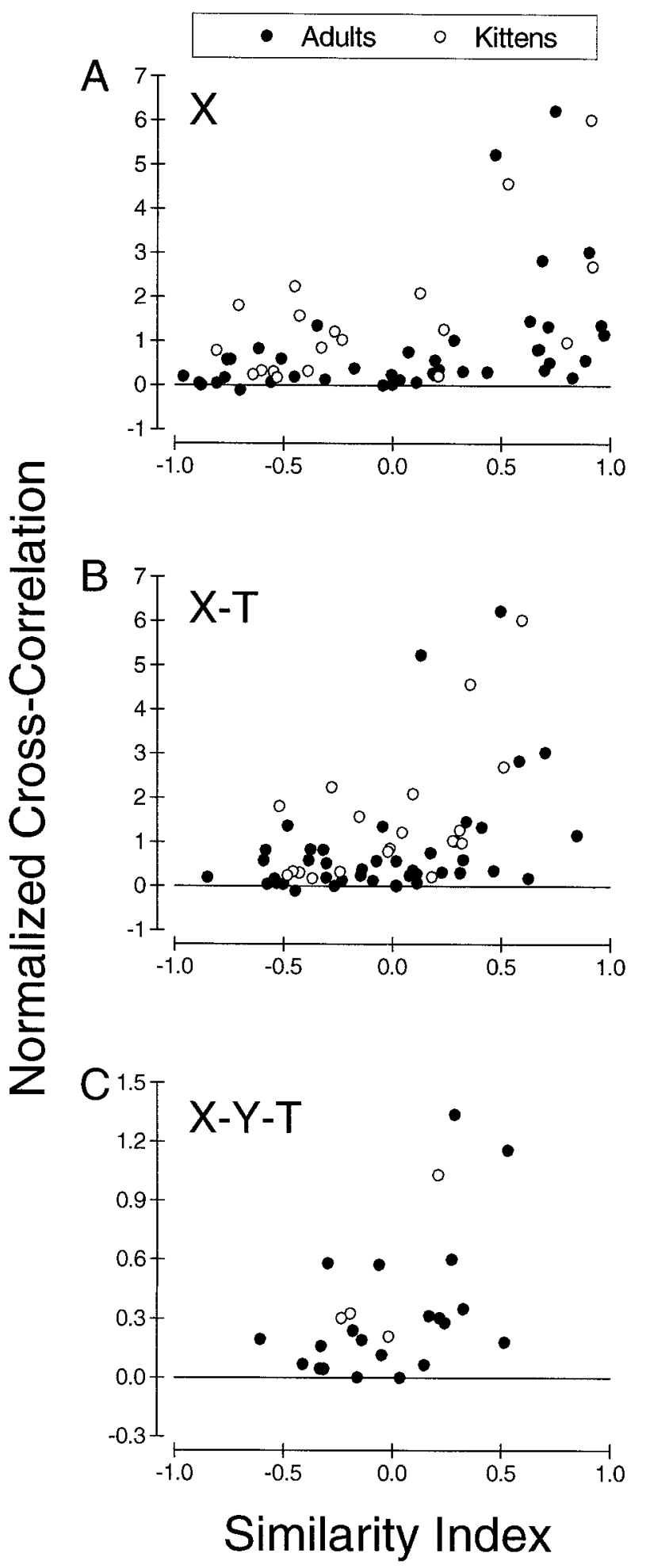

Figure 6. Relationship between correlated discharge and receptive field similarity. Each graph plots normalized cross-correlation (see Results for details) against the similarity index computed from a different RF cross section: $X(A), X-T(B)$, and $X-Y-T(C)$. Filled circles show data from adult cats; open circles show data from 4 -week-old kittens. Note that the magnitude of correlated discharge is larger in the $\mathrm{SI}_{X}$ and $\mathrm{SI}_{X-T}$ distributions. This is because these distributions include 1-D reverse correlation runs in which elongated bars are used. These bars are a more effective stimulus than the smaller bars used in 2-D runs, and therefore elicit both stronger individual responses and stronger correlated responses.

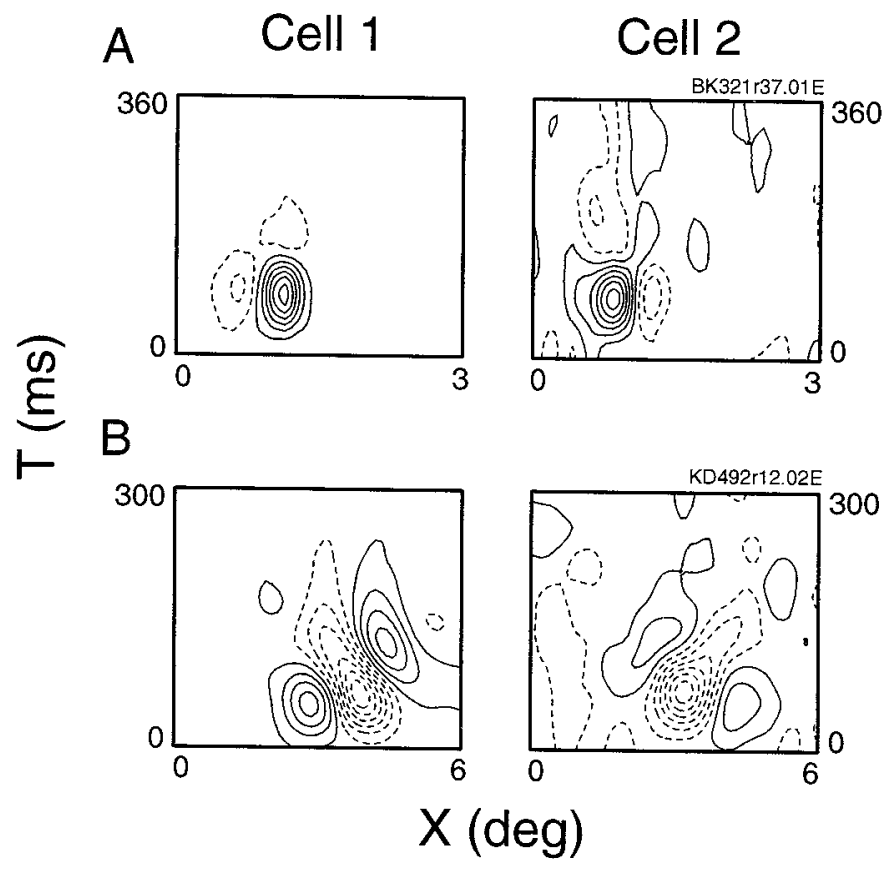

Figure 7. Examples of simple cell pairs that have SI values near zero in the $X-T$ domain. $A$, The $X-T$ profiles of this pair of simple cells are similar, except for a spatial phase difference of $\sim 90^{\circ}$ (i.e., spatial quadrature); $\mathrm{SI}_{X-T}=0.11 . B$, The two members of this pair of cells prefer opposite directions of motion, as evidenced by the difference in the space-time orientation of their subregions. $\mathrm{SI}_{X-T}$ for this pair is -0.13 .

$$
G(x)=\operatorname{Exp}\left(-\left(\frac{2\left(X-X_{0}\right)}{w}\right)^{2}\right) \operatorname{Cos}\left(2 \pi s f\left(X-X_{0}\right)+P\right),
$$

where $X_{0}, w, s f$, and $P$ are free parameters. $X_{0}$ and $w$ represent the center position and width, respectively, of the Gaussian RF envelope; $s f$ and $P$ correspond to the spatial frequency and phase of the sinusoid. The Gabor function has been shown to provide good fits to the spatial RF profiles of simple cells, and is attractive because it has physiologically meaningful parameters (for example, see Field and Tolhurst, 1986; Jones and Palmer, 1987; DeAngelis et al., 1993a). The spatial profiles of the two separable components of the model differ in phase by $90^{\circ}$ (i.e., $P_{1}=P_{2}+$ $90^{\circ}$ ). Thus, the two subunits are said to be in spatial quadrature.

The temporal waveform, $\mathrm{H}(T)$, of each separable subunit is also modeled as a Gabor function, but is temporally skewed. This skewing is necessary to account for the observation that temporal response profiles of simple cells typically have a fast rising phase, a slower decaying phase, and unequally spaced zero crossings (see DeAngelis et al., 1993a for details). Thus, the temporal waveforms cannot be modeled adequately with a simple periodic function. In our formulation, the temporal profile is a Gabor function in a skewed time frame:

$$
H(t)=\operatorname{Exp}\left(-\left(\frac{2\left(T_{S}-T_{0}\right)}{c}\right)^{2}\right) \operatorname{Cos}\left(2 \pi t f\left(T_{S}-T_{0}\right)+Q\right),
$$

where the skewed time coordinate, $T_{\mathrm{s}}$, is given by $T_{\mathrm{s}}=2 \arctan$ $(\beta \mathrm{T}) / \pi$. For simplicity, the time-skewing function is chosen to be the arctan function, which is desirable because it has only one free parameter, $\beta$. Note, however, that this choice is quite arbitrary; other sigmoidal functions would also be suitable. The remaining 


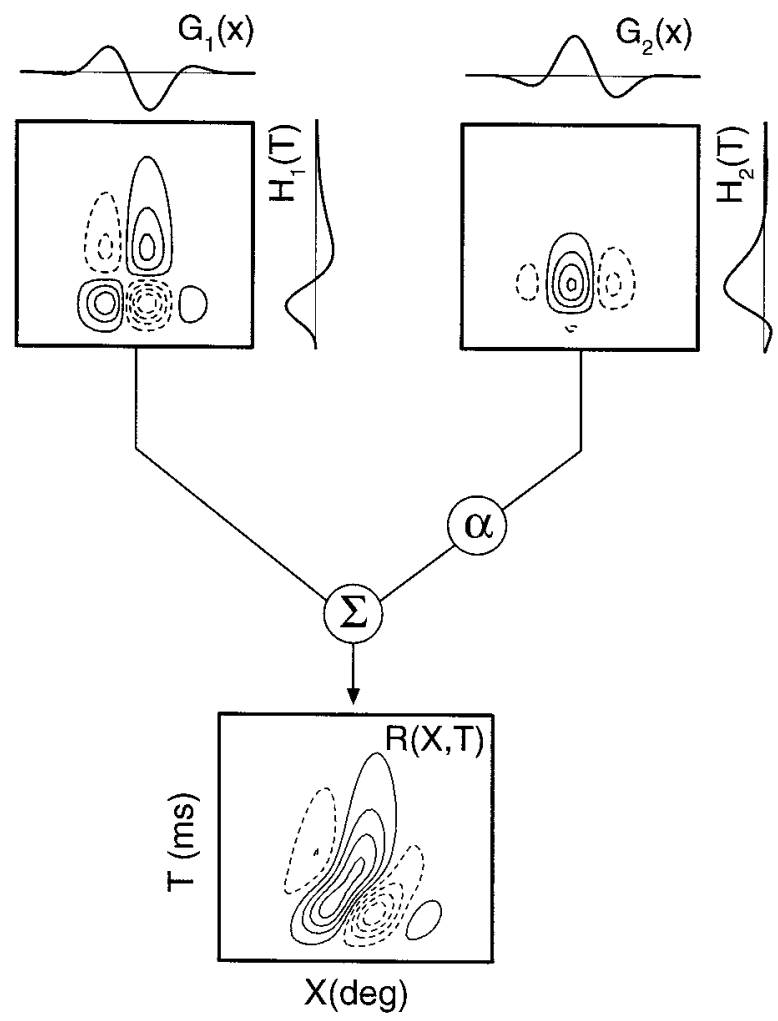

Figure 8. Spatiotemporal RF model used to fit the $X-T$ profiles of simple cells in this study. In this model, a spatiotemporally inseparable RF (bottom) is constructed as the weighted sum of two separable RFs. The two separable subunits are identical except for a $90^{\circ}$ difference in their spatial and temporal phases (see Results for details). As the weight, $\alpha$, on the second subunit increases from 0.0 to 1.0 , the resultant model RF changes from space-time separable to strongly inseparable. $G_{1}(X)$, $H_{1}(T), G_{2}(X), H_{2}(T)$, and $R(X, T)$ denote the quantities referred to in Equation 2 (see Results).

parameters of the temporal Gabor function have definitions analogous to those of the spatial Gabor in Equation 3.

The RF model of Figure 8 is very similar in structure to models proposed previously (Adelson and Bergen, 1985; Watson and Ahumada, 1985), the main difference being the exact formulation of the temporal response profile. A detailed discussion of the design and biological plausibility of the model is beyond the scope of the present paper and will be the subject of a future publication. For the present purpose, it is sufficient to note that the model has meaningful parameters and provides good fits to the RF profiles of simple cells.

Figure 9 shows the best fits of the model to the $X-T$ profiles of three pairs of simple cells. We have quantified the goodness of fit by computing a fractional error metric, which is defined as the sum squared error divided by the sum of squares of the data. The examples shown in Figure 9 were chosen to illustrate the quality of fits associated with fractional errors of different magnitudes. For the pair of cells in Figure 9A, the fractional errors are 0.062 and 0.046 (top and bottom, respectively); these were among the better fits that we obtained. The pair of cells in Figure $9 B$ exhibit average quality fits, with fractional error values of 0.144 and 0.126 (top and bottom, respectively). Last, Figure $9 C$ shows a pair of fits that have fractional errors (0.195 and 0.254, top and bottom, respectively) among the largest in our sample. Nevertheless, the error profiles are quite unstructured for these cells, as well as those in panels $A$ and $B$. Thus, although the measured RF profiles are quite noisy in Figure $9 C$, the model captures the basic structure of the RFs quite effectively.

The distribution of the fractional error metric for all simple cells studied is shown in Figure 10. The mean values are 0.16 for adult cats and 0.21 for kittens. The average fractional error for kittens cells is significantly larger $(t=3.06 ; p=0.003)$ than that for adults, which presumably reflects the fact that the data from kittens are somewhat noisier (because of weaker responses).

It should be noted that we have only fit the $X-T$ profiles of simple cells and not the full $X-Y-T$ profiles. This was done mainly because $X-Y-T$ data were only measured for 29 of 45 pairs of cells from adults and 4 of 21 pairs from kittens. Given that the RFs of nearby pairs of simple cells are very similar along the $Y$ dimension (Fig. 3), however, we do not sacrifice much by neglecting the $Y$ dimension in the following analyses.

\section{Clustering of receptive field parameters}

Which aspects of receptive field structure are clustered within primary visual cortex? To address this question, we compared the values of various receptive field parameters for each pair of simple cells studied. Figure 11 shows the raw data that were used in these comparisons. Each panel in Figure 11 is a scatter plot showing the value of a particular RF parameter for one neuron (cell 2, vertical axis) plotted against the corresponding value for a simultaneously recorded, nearby neuron (cell 1 , horizontal axis). As expected from the orderly map of orientation found in striate cortex (Hubel and Wiesel, 1974; Blasdel, 1992), preferred orientation is strongly clustered, with most data points tightly grouped around a diagonal line of unity slope (Fig. 11 $A$ ). Clustering is also readily apparent, although somewhat weaker, for RF width (Fig. 11C) and preferred spatial frequency (Fig. 11E). Temporal parameters of the receptive field, peak response latency (Fig. $11 B$ ), response duration (Fig. 11D), and preferred temporal frequency (Fig. $11 F$ ), also exhibit a modest, but significant (as described below), degree of clustering. In contrast, spatial phase (Fig. 11G) is scattered within the dashed line boundaries. Because this variable is circular, the largest possible phase difference between a pair of RFs is $180^{\circ}$; thus, all of the data points in Figure $11 G$ are constrained to fall between the dashed lines. This restriction also applies to the temporal phase data (Fig. $11 \mathrm{H})$, which are plotted on the same scale as spatial phase to facilitate comparison. Whereas the spatial phases are spread uniformly throughout the range of possible values, the temporal phases are confined to a narrow range. These observations are consistent with the similarity index data of Figures 3 and 4, which show that there is much more cell-to-cell variation in RF shape along the $X$ dimension than along the $T$ dimension. Last, Figure $11 I$ shows that there is no apparent clustering for the direction index, $\alpha$. There is, however, a clear tendency for nearby neurons to have the same preferred direction of motion (41 of 66 pairs in the top right and bottom left quadrants), rather than opposite preferred directions (25 of 66 pairs in the other quadrants). This tendency for nearby cells to have the same preferred direction is consistent with previous single-unit studies in areas 17 and 18 (Payne et al., 1980; Tolhurst et al., 1981; Berman et al., 1987).

To quantify the degree of clustering for each parameter, as well as its statistical significance, we performed a permutation test as follows. First, a distribution of the absolute pairwise differences was constructed for each RF parameter in Figure 11 (i.e., the data were collapsed onto an axis perpendicular to the diagonal, and the resulting distribution was then folded in half around zero). The median value of this distribution will be referred to as the 
A
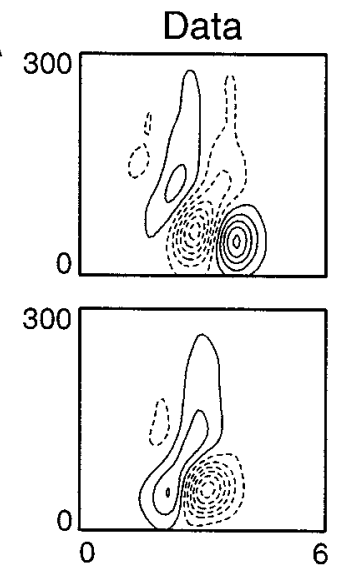

B

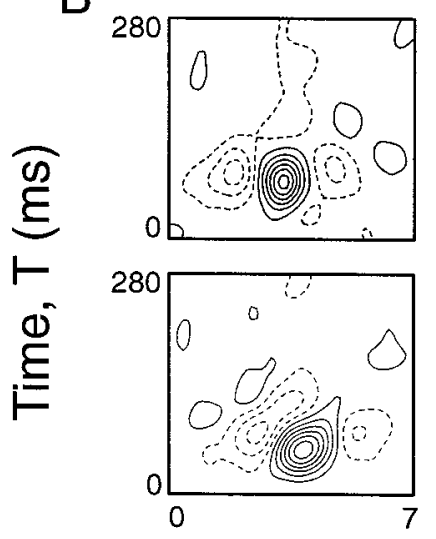

C
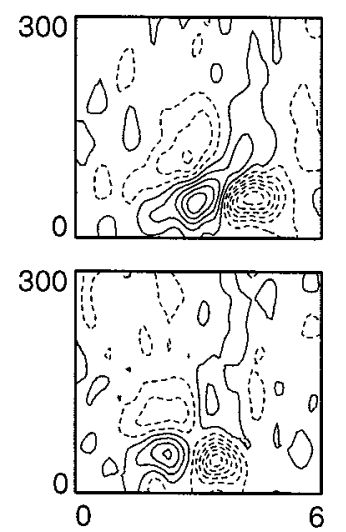

Fit
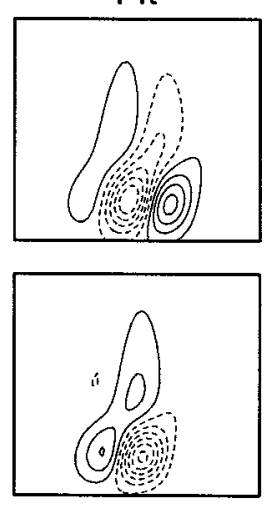

$$
\text { . }
$$
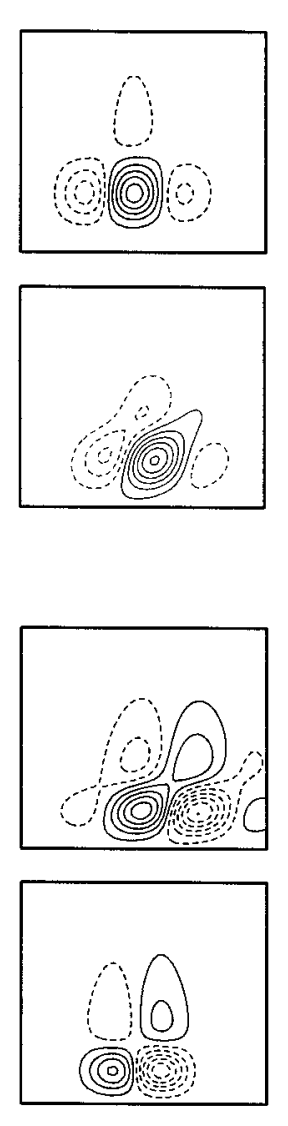

Space, X (deg)
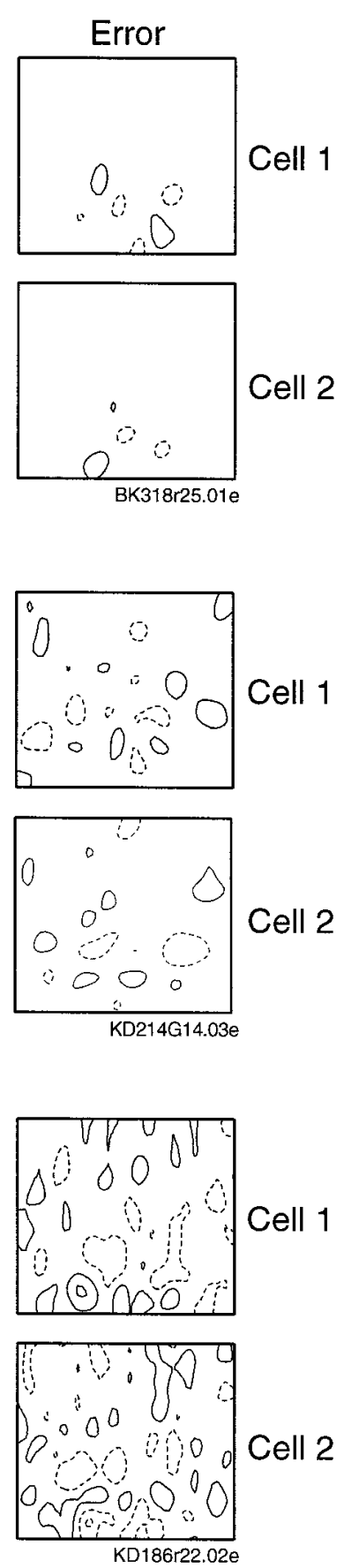

KD186r22.02e

Figure 9. Representative fits of the spatiotemporal $\mathrm{RF}$ model to $X-T$ profiles for three pairs of simple cells $(A-C)$. For each neuron, the left panel shows the measured RF profile, the center panel shows the best fit of the model depicted in Figure 8, and the right panel shows the error profile (i.e., the difference between the data and the fit). Note that all three contour maps for a given cell are plotted on the same response scale. See Results for additional details.

paired median. We then constructed an artificial distribution of pairwise differences by drawing random pairings from the overall sample of neurons, and we computed the median of this random sample (the random sample was the same size as the originalsample of pairs). This process was repeated 5000 times, and we computed the median of the distribution of random medians, which we refer to as the grand random median. A clustering index is then defined as the ratio of the grand random median to the paired median. The larger this ratio, the stronger the clustering. Figure 12 shows values of the clustering index for each RF parameter. Asterisks above some bars in Figure 12 indicate that the clustering index for these parameters is significantly $>1.0$ $\left({ }^{*} p<0.01 ;{ }^{*} p<0.05\right)$. Statistical significance is determined by the proportion of simulations in which the random sampling of pairs had a median value less than that of the actual paired data (e.g., $p=0.01$ indicates that the random median was less than the paired median in 50 of 5000 simulations).

The data of Figure 12 indicate that the degree of clustering for preferred orientation far exceeds that for any other RF parameter. Nevertheless, both cats and kittens exhibit highly significant clustering for RF width $(w)$ and preferred spatial frequency $(s f)$. We also observe statistically significant, but weaker, clustering for 


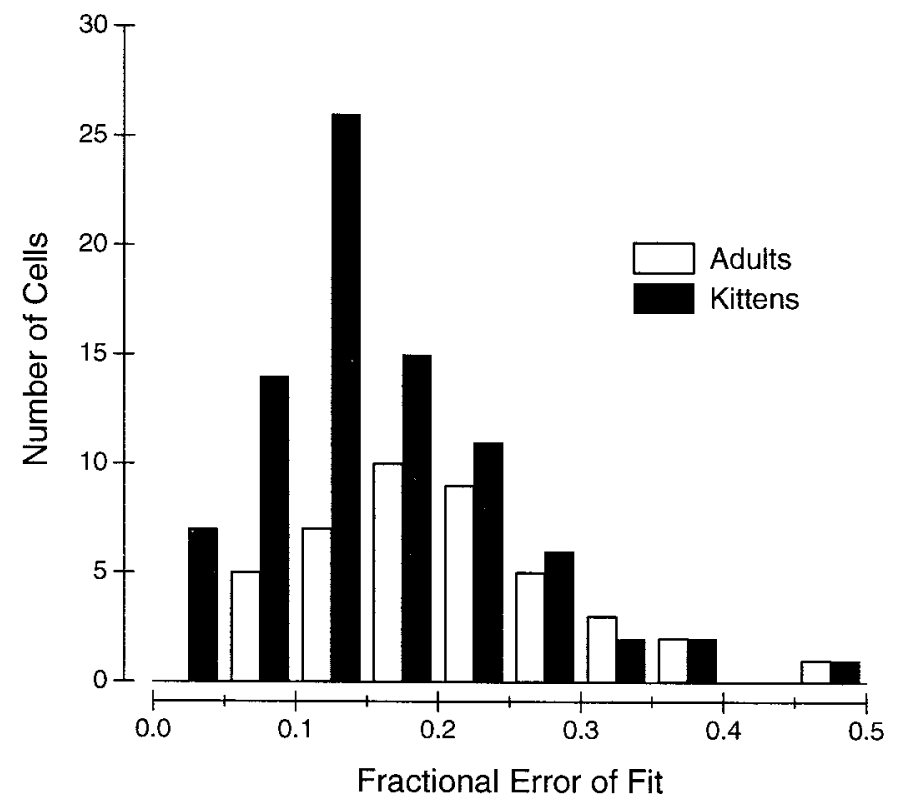

Figure 10. Distribution of the fractional error metric used to quantify the quality of fits of the RF model to data. Fractional error of the fit is defined as the sum of squares of the error profile (Fig. 9A, right) divided by the sum of squares of the raw data (Fig. $9 A$, left $)$. Filled and open bars denote data from adult cats and kittens, respectively.

all three of the major temporal response parameters: latency $\left(t_{0}\right)$, duration $(D)$, and preferred temporal frequency $(t f)$. Parameters of RF shape, namely the spatial phase $(P)$ and temporal phase $(Q)$, do not exhibit any significant clustering. Finally, there is no significant clustering of the direction index $(\alpha)$. This finding is consistent with recent optical imaging studies, which give little indication of direction clustering in cat area 17 (Bonhoeffer et al., 1995).

A note of caution should be added here regarding interpretation of clustering indices. A small clustering index could arise either because there are random variations from cell to cell in a particular parameter (e.g., spatial phase) or because there is little variation in a given parameter from cell to cell (e.g., temporal phase) in absolute terms. Thus, there is no discrepancy between the observation that temporal RF parameters exhibit generally weak clustering (Fig. 12) and the observation that temporal RF profiles tend to be highly similar between pairs of neighboring simple cells (Figs. 3, 4).

Before concluding that all of the correlations analyzed in Figures 11 and 12 are genuine, we must consider the possibility that some of these correlations result from intervening variables. For example, the observed correlation between RF widths in Figure $11 C$ could be produced by a strong correlation between RF width and eccentricity across the population of cells or by a strong correlation between RF width and preferred spatial frequency. To address this possibility, we performed a series of multiple regression analyses. In one of these analyses, we chose the RF width of cell 1 as the dependent variable, and as independent variables, we chose the RF width of cell 2, the preferred spatial frequency of cell 1 , and the average eccentricity of the cell pair (the two members of a pair were seldom separated by $>1^{\circ}$; Fig. 13A). This model allows us to examine the partial correlation between pairwise RF widths in the presence of variations in spatial frequency and eccentricity. The results show that the pairwise correlation between RF widths is not driven by the other variables (adults: partial $r=0.54, p<0.001$; kittens: partial $r=$ $0.76, p<0.001)$. Thus, we conclude that the observed correlation between RF widths (Fig. 11C) is real. It is also worth noting that there was a marginally significant partial correlation between RF width and eccentricity for adults (partial $r=0.29 ; p=0.056$ ). We were somewhat surprised that this correlation was so weak in light of the general trend for RF size to increase with eccentricity in various cortical areas, but this result is consistent with previous findings for simple cells (Wilson and Sherman, 1976). It should also be noted, however, that some of our eccentricity estimates were unfortunately quite crude; thus, noise in our eccentricity data may have masked a somewhat stronger correlation. Specifically, our eccentricities ranged from 3 to $15^{\circ}$, and we estimate that most of our measurements were accurate to within 2 or $3^{\circ}$.

Using a similar analysis, we also tested whether the observed pairwise correlation in preferred spatial frequency (Fig. 11E) held up in the presence of variations in RF width and eccentricity. Again, the pairwise correlation (cell 1 vs cell 2 for $s f$ ) remained highly significant (adults: partial $r=0.68, p<0.0001$; kittens: partial $r=0.83, p<0.0001$ ). Thus, the correlation evident in Figure $11 E$ also appears to be genuine.

Finally, we also performed similar analyses to test whether pairwise correlations in response latency (Fig. 11B), response duration (Fig. 11D), and preferred temporal frequency (Fig. $11 F$ ) held up to inclusion of eccentricity and the other temporal parameters. Briefly, we found that, for adults, the partial correlations for latency $\left(t_{0}\right)$ and temporal frequency $(t f)$ were significant ( $p<0.05$ and $p<0.001$, respectively), whereas the partial correlation for duration $(D)$ was not $(p>0.2)$. In contrast, for kittens, we found that the partial correlation for duration was significant $(p<0.001)$, but the partial correlations for latency and temporal frequency were not $(p>0.1)$. Note that this pattern of results is similar to that seen in Figure 12. For adults, clustering indices for latency and temporal frequency were more significant $\left({ }^{*} p<0.01\right)$ than the index for duration $(* p<0.05)$. For kittens, the clustering index for duration was more significant $(* * p<$ $0.01)$ than those for latency and temporal frequency $(* p<0.05)$. Thus, to summarize, all of the parameters that are indicated by double asterisks in Figure 12 exhibited pairwise correlations that held up in our multiple regression analyses.

\section{Analysis of receptive field overlap}

The clustering analyses of the previous section did not include the RF position variable $x_{0}$ because RF position in our model only refers to the location of the center of the RF within the reversecorrelation mapping grid; it does not refer to absolute retinotopic position. Nevertheless, our model does provide a quantitative measure of the positional offset (along the $X$-axis) between RFs of nearby neurons. Thus, this information bears on the issue of RF overlap.

Figure $13 A$ shows the distribution of positional offsets between RFs of all pairs of simple cells recorded in this study. Nearby simple cells were rarely found to have RFs separated by $>1^{\circ}$. More related to the issue of RF overlap, Figure $13 B$ shows the distribution of positional offsets normalized by the average RF width of each pair of neurons. For the vast majority of cell pairs, the two RFs are offset by less than one-quarter of the width of the receptive fields. Thus, RFs of nearby simple cells overlap extensively. This is consistent with data reported recently for vertical penetrations in area 17 (Das and Gilbert, 1997). Finally, Figure $13 C$ shows positional offset expressed as a number of cycles at the preferred spatial frequency of the cells. For most pairs, the RF 

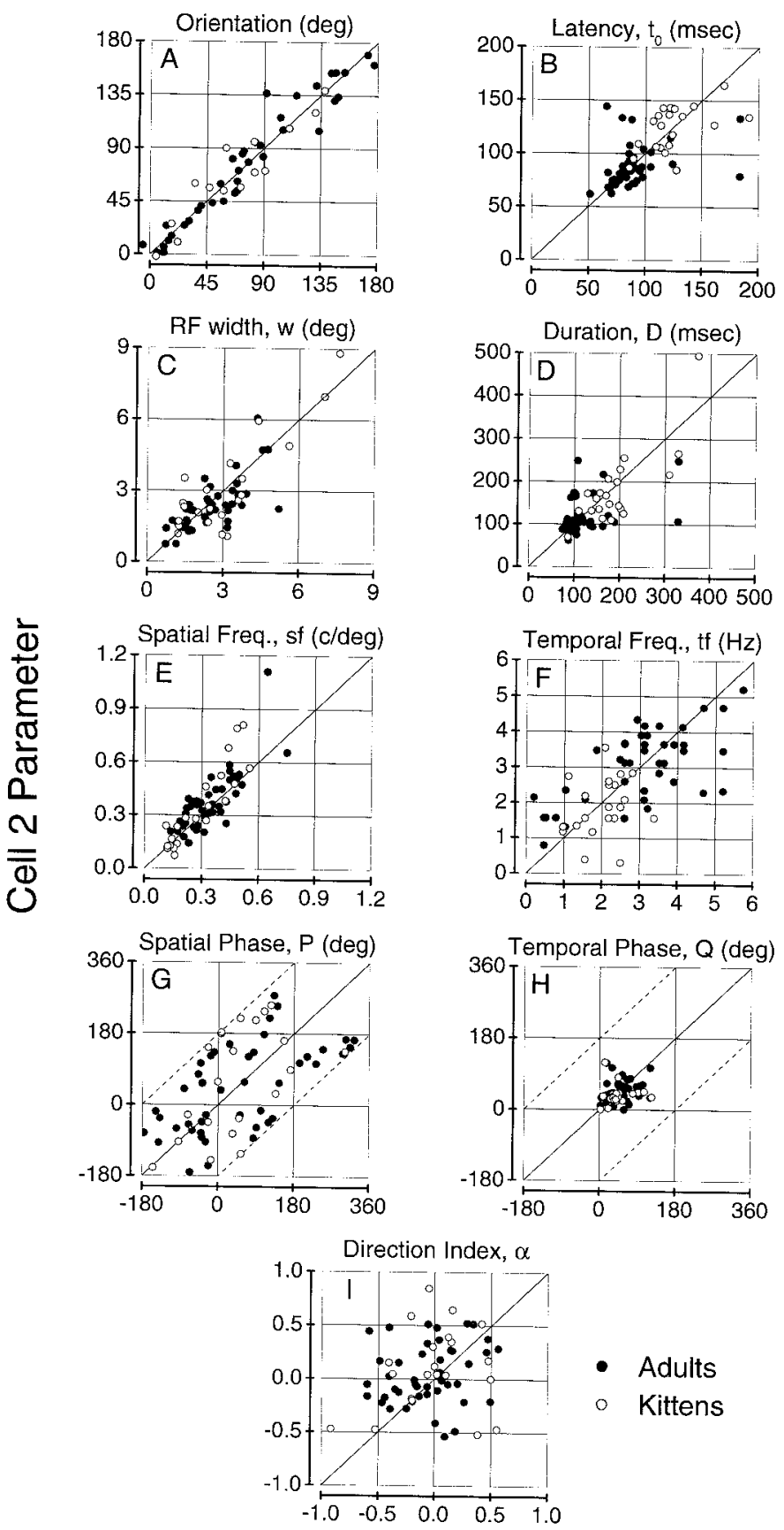

Cell 1 Parameter

Figure 11. Summary of pairwise correlations in various RF parameters. Each panel here is a scatter plot in which the parameter value for one cell (cell 1) is plotted on the horizontal axis, and the value for a simultaneously recorded neuron (cell 2) is plotted on the vertical axis. Filled and open circles represent data from adult cats $(n=45$ pairs) and kittens $(n=$ 21 pairs), respectively. $A$, Preferred orientation shows strong clustering, with most data points distributed tightly around the diagonal line of unity slope. Note that these data were obtained from responses to drifting grating stimuli, whereas the remaining data in this figure were extracted from fits to the $X-T$ profiles. $B$, Peak response latency, $t_{0}$, shows modest clustering. Note that $t_{0}$ here is expressed in units of milliseconds, whereas $T_{0}$ in the model (Eq. 4) is defined in skewed time coordinates. $C$, Scatter plot of RF width, $w$, as defined in Equation 3. D, Scatter plot of response duration, $D$. $D$ is defined as the width of the temporal response envelope at a criterion amplitude of $1 / \mathrm{e}$ (or 0.37 of the peak amplitude), and is expressed in units of milliseconds. Note that, although $D$ is determined by the parameter $c$ in Equation 4, $c$ is not used directly here because it is offset is less than one-quarter cycle (or $90^{\circ}$ ) of phase. Note that the measurement procedure we used for the majority of cells does not provide sufficient data to allow analysis of the $Y$ dimension.

\section{Relative contribution of model parameters to pairwise differences in RF organization}

In the previous sections, we have shown that different attributes of RF structure cluster to varying degrees within striate cortex. Here, we describe a quantitative method for determining how each parameter contributes to the overall difference in RF structure between two neurons. Because different parameters of the RF model (Eqs. 2-4) have different units of measure, it is difficult to assess the relative contributions of different parameters from the data of Figure 11. It would be difficult to state, for example, whether a difference in RF width of $0.5^{\circ}$ is more or less substantial than a difference in temporal phase of $45^{\circ}$. Thus, we need to transform the pairwise differences in each RF parameter into a common metric. We have chosen to do this as follows. First, we fit the $X-T$ data from a pair of simple cells simultaneously and determine the total error of the joint fit. Because the formulation of Equations 2-4 has 11 free parameters (for each cell), this corresponds to a 22 parameter fit (note, however, that the optimal solution to the 22 parameter fit is the same as the optimal solutions to the two 11 parameter fits, done separately.). Next, we choose one of the 11 different parameters of the model (e.g., $x_{0}$ ), and we force it to have a common value (which is free to vary) for the two members of the pair (thus yielding a 21 parameter fit). We repeat this fitting procedure with each different parameter of the model held as the common parameter. We then compute, for each different common parameter, a measure of the increase in the total error of the fit:

$$
\% \text { error elevation }=\frac{E_{21}-E_{22}}{E_{22}},
$$

where $E_{21}$ is the error of the simultaneous fit when one parameter value is held common to the pair, and $E_{22}$ is the total error when all 22 parameters are free to vary individually.

Figure 14 illustrates the results of this analysis for a pair of simple cells that differ markedly in spatial phase. Figure $14 \mathrm{~A}$ shows the best fit of the model to both RF profiles when all 22 parameters are free to vary. The bar graph of Figure $14 B$ shows the percent error elevation when each different parameter of the RF model is forced to have a common value for the pair. For all parameters other than spatial phase, $P$, there is little increase in the error of the joint fit. When $P$ is the common parameter, however, the error of the fit nearly doubles, as can be seen by inspection of the fit and error profiles in Figure 14B (inset). Thus,

\section{$\leftarrow$}

defined in skewed time coordinates. $E$, Preferred spatial frequency, $s f$ (defined as in Eq. 3), shows pronounced clustering. F, Scatter plot of preferred temporal frequency, $t f$. Note that $t f$ here is expressed in units of hertz. $G$, Scatter plot of the spatial phase of the receptive field, $P$. Because $P$ is a circular variable, the largest possible difference in phase between two neurons is $180^{\circ}$. Thus, all of the data points shown here are constrained to fall within the pair of dashed lines. $H$, Distribution of temporal phase, $Q$. These data are plotted on the same axes as those of panel $G$ to facilitate comparison. I, Scatter plot for the linear direction selectivity index, $\alpha$. Positive and negative values of $\alpha$ correspond to opposite directions of motion, and larger values of $\alpha$ correspond to stronger direction selectivity. Thus, data points in the top right quadrant denote pairs of neurons with the same direction preference, whereas points in the top left or bottom right quadrants indicate pairs with opposite preferred directions of motion. 


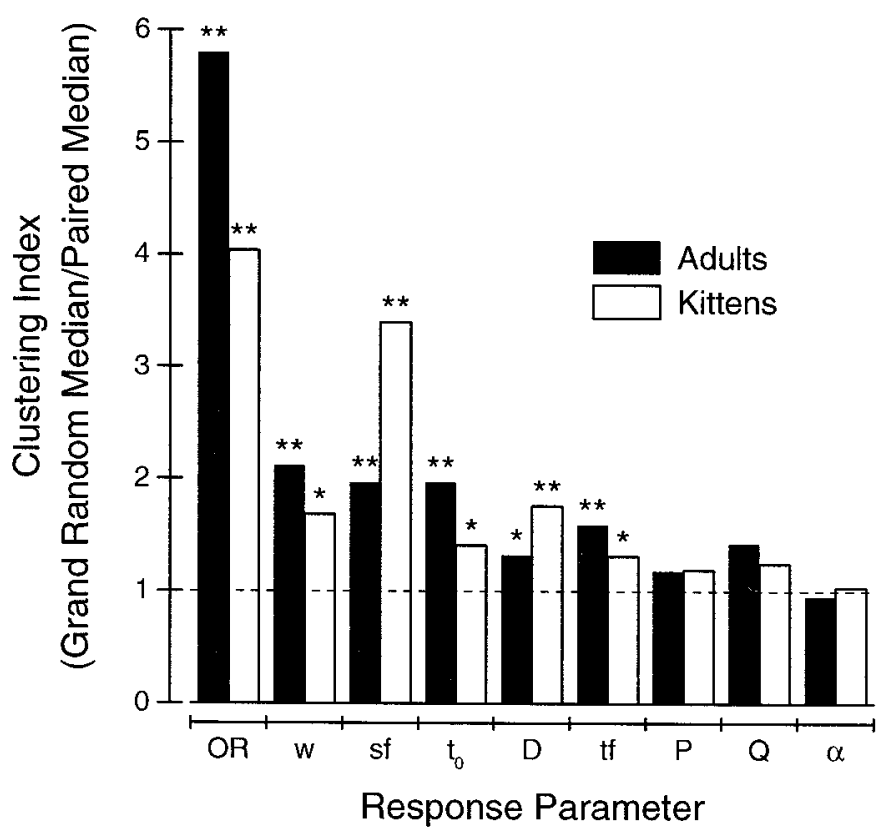

Figure 12. Quantitative analysis of clustering for the spatial and temporal response parameters of Figure 11. Filled and unfilled bars correspond to data from adult cats and kittens, respectively. Each bar gives the clustering index (see Results for details) for a particular response parameter. Asterisks above some bars indicate that the associated clustering index is significantly $>1.0\left({ }^{* *} p<0.01 ;{ }^{*} p<0.05\right)$. OR, Preferred orientation; $w$, RF width; $s f$, preferred spatial frequency; $t_{0}$, peak response latency; $D$, response duration; $t$, preferred temporal frequency; $P$, spatial phase; $Q$, temporal phase; and $\alpha$, direction selectivity index.

for this pair of simple cells, spatial phase accounts for nearly all of the difference in RF structure.

Figure $15 A$ summarizes the results of this analysis for all pairs of simple cells recorded from adult cats and kittens. In this graph, the mean percent error elevation $( \pm$ SE) across all pairs of cells is plotted for each RF parameter held common to the pair. Clearly, spatial phase $(P)$ is the single largest contributor to RF differences between pairs of adjacent neurons, producing an average error elevation of $25.6 \%$ for adults and $14.3 \%$ for kittens. The second largest contributor is RF position $\left(x_{0}\right)$, which increased the error of the fits by an average of $14 \%$ in adults and $6.7 \%$ in kittens, when chosen as the common parameter. Other than a modest contribution from the direction selectivity index, $\alpha$, the remaining RF parameters contribute very little to the overall difference between nearby RFs. Note that this finding is consistent with the similarity index data of Figures 3 and 4, which indicate that most RF differences originate along the $x$ dimension.

The average error elevations for adult cell pairs are generally larger than those for kittens; however, this could be caused by the fact that the $X-T$ data from kittens are generally noisier (Fig. 10). To test this possibility, we performed an ANCOVA with the following factors: age, common parameter, fractional error of the joint (22 parameter) fit, and all second-order interaction terms. There is a significant interaction $(p=0.02)$ between age and fractional error. When taken into account, the main effect of age becomes marginally significant $(p=0.04)$. Thus, differences in percent error elevation between adults and kittens should be interpreted with caution. Nevertheless, the pattern of results in Figure $15 A$ is quite similar for adults and kittens, as evidenced by

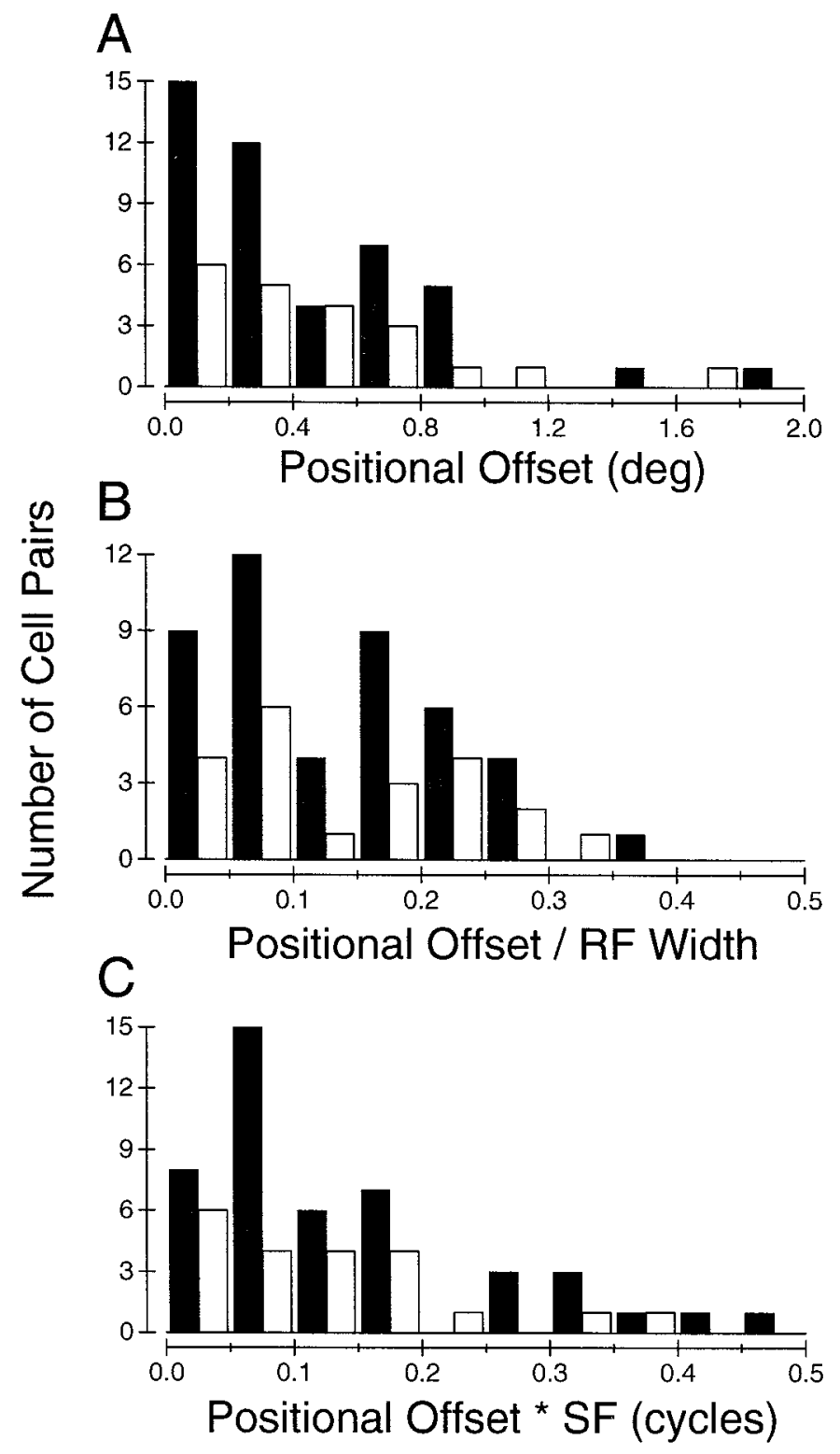

Figure 13. Analysis of receptive-field overlap. A, Distribution of positional offsets between RFs of simultaneously recorded pairs of simple cells. Filled and unfilled bars show data from adult cats and kittens, respectively. Positional offset is defined as the spatial displacement (along the $X$-axis) between the centers of the spatial envelopes of two RFs [i.e., $\left.\operatorname{abs}\left(x_{0 \mathrm{~A}}-x_{0 \mathrm{~B}}\right)\right] . B$, Distribution of positional offsets normalized by the average RF width of the two neurons [i.e., $\operatorname{abs}\left(x_{0 \mathrm{~A}}-x_{0 \mathrm{~B}}\right) /\left(0.5\left(w_{\mathrm{A}}+\right.\right.$ $\left.w_{\mathrm{B}}\right)$ ], where the subscripts $A$ and $B$ denote the two members of a pair of neurons. $C$, Distribution of positional offsets expressed as a number of cycles at the preferred spatial frequency of the cells. The offset in cycles is computed as $\operatorname{abs}\left(x_{0 \mathrm{~A}}-x_{0 \mathrm{~B}}\right)\left(0.5\left(s f_{\mathrm{A}}+s f_{\mathrm{B}}\right)\right.$, where $s f_{\mathrm{A}}$ and $s f_{\mathrm{B}}$ are the preferred spatial frequencies for the two neurons of each pair.

the lack of a significant interaction term between age and common parameter $(p=0.97)$.

The relative contributions of spatial phase and position are compared on a pair-by-pair basis in Figure $15 B$. There is no significant correlation $(r=0.26, p=0.08$ for adults; $r=$ $-0.03, p=0.98$ for kittens) between these two factors. Some pairs of neurons differ almost exclusively in terms of either phase (data near vertical axis) or position (data near horizon- 

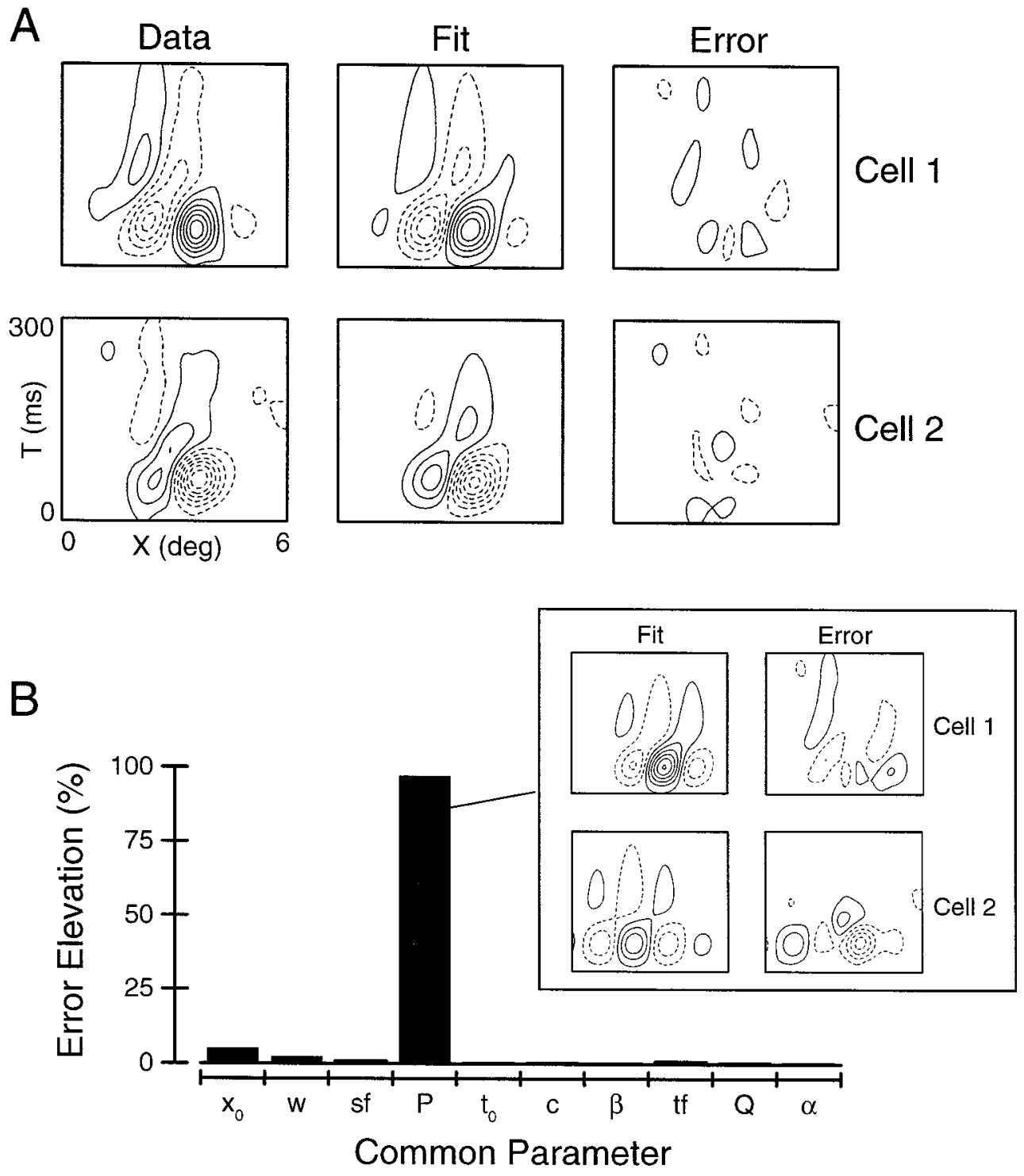

Figure 14. Simultaneous-fitting analysis for quantifying the contribution of various RF parameters to the overall difference in structure between a pair of RFs. $A$, Data, fit, and error profiles for a pair of simple cells from an adult cat that differ predominantly in terms of spatial phase (i.e., an antiphase pair). The $X-T$ data for both neurons was fit simultaneously with the RF model of Equations 2-4 (22 parameter fit). B, Summary of changes in the overall error of the fit, expressed as percent error elevation, when different parameters of the RF model are forced to have a common value for the two neurons. Because one parameter is common to the pair, the resulting fit has 21 parameters. The inset shows the best fit of the model, along with the resulting error profiles, when spatial phase is the common parameter. Note that the error profiles are larger and substantially more structured than those in panel $A$. Parameters of the model are denoted as follows: $x_{0}, \mathrm{RF}$ center position; $w$, RF width; $s f$, preferred spatial frequency; $P$, spatial phase; $t_{0}$, peak response latency; $c$, temporal envelope width; $\beta$, temporal skewing factor; $t f$, preferred temporal frequency; $Q$, temporal phase; and $\alpha$, direction selectivity index.

tal axis), whereas other pairs of cells differ substantially in both respects.

\section{Spatial phase relationships: quadrature and antiphase}

It has previously been reported (Pollen and Ronner, 1981; Liu et al., 1992) that the RFs of nearby simple cells tend to differ in spatial phase by either $90^{\circ}$ (quadrature phase) or $180^{\circ}$ (antiphase). To be considered as a true quadrature or antiphase pair, however, two neurons must differ only in spatial phase, without differing substantially in other parameters such as size or spatial frequency. One advantage of our simultaneous-fitting analysis is that it allows us to establish rigorous criteria for selecting pairs of neurons that differ only in spatial phase (or any other parameter).

Figure $16 \mathrm{~A}$ plots the relative contribution of spatial phase to the overall difference between pairs of RFs as a function of the spatial phase difference between each pair. Relative contribution is defined as the percent error elevation caused by spatial phase, divided by the total percent error elevation caused by all parameters (e.g., the relative contribution of spatial phase is 0.90 for the pair of cells in Fig. 14B). Not surprisingly, the relative contribution of spatial phase generally increases with the magnitude of the spatial phase difference $(r=0.58 ; p<0.0001)$. Filled symbols in
Figure $16 A$ denote pairs of simple cells that differ predominantly in terms of spatial phase. Our criterion for selecting these neurons is that the relative contribution of each parameter, other than spatial phase, does not exceed 0.20 . Note that this criterion does include pairs of neurons that have spatial phase differences near zero; such pairs of cells have $X-T$ receptive field profiles that are essentially identical in shape.

Figure $16 B$ shows the distribution of spatial phase differences for all pairs of simple cells that meet the criterion described above (here and in Fig. 16C, filled and unfilled bars are for cats and kittens, respectively). Clearly, the spatial phase relationships between nearby pairs of simple cells are not limited solely to quadrature $\left(90^{\circ}\right)$ and antiphase $\left(180^{\circ}\right)$ conditions. Although such pairs certainly do exist, there are also several pairs of neurons with other phase differences, including $\sim 0^{\circ}$. The distribution of phase differences is not uniform $\left(\chi^{2}=10.8\right.$; df $=4$; $p=0.028$; data pooled across cats and kittens, $n=35$ ), however, and there are many more pairs of neurons with phase differences in the range from 90 to $180^{\circ}(n=26)$ than there are in the range from 0 to $90^{\circ}(n=9)$. In contrast to Figure $16 B$, the distribution of spatial phase differences is indistinguishable from uniform $\left(\chi^{2}=\right.$ 

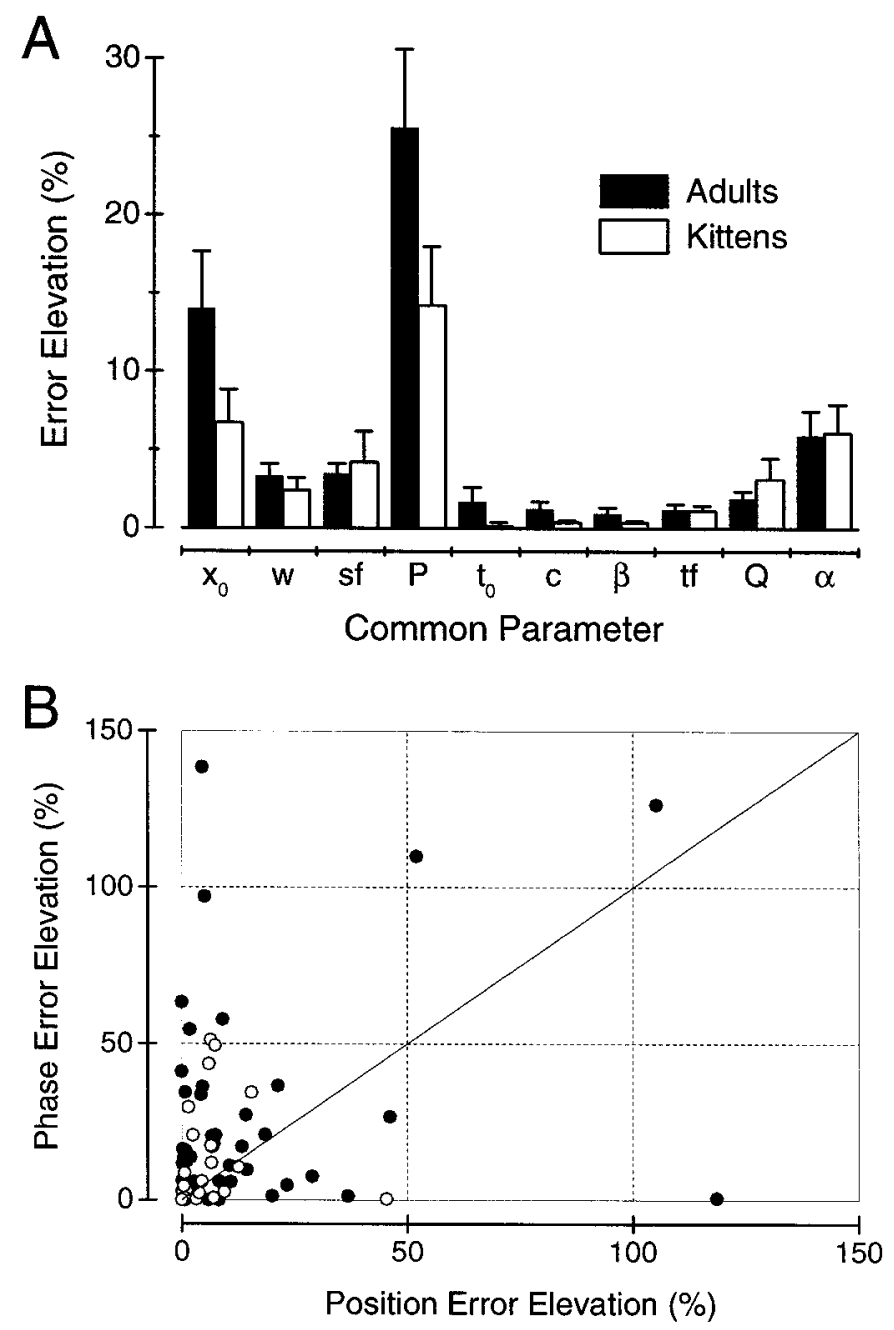

Figure 15. Summary of the relative contributions of various model parameters to the overall difference in structure between pairs of RFs. $A$, Mean error elevation $(+1 \mathrm{SE})$ is shown for each RF parameter. Filled and open bars show data from adult cats and kittens, respectively. Parameter notations are as described in Figure 14B. B, Phase error elevation is plotted against position error elevation for all pairs of simple cells from adults ( filled circles) and kittens (open circles).

1.3; $\mathrm{df}=4 ; p=0.86$; pooled data, $n=31$ ) for pairs of simple cells that differ in parameters other than spatial phase (Fig. 16C).

\section{Binocular simple-cell pairs}

Thus far, we have only made monocular comparisons between RFs of neighboring simple cells. Although our $X-T$ and $X-Y-T$ profiles provide a fairly complete description of the monocular response properties of each neuron, we have neglected a stimulus dimension, binocular disparity, that may also contribute substantially to the similarity or dissimilarity between RFs of neighboring neurons. We have previously shown (DeAngelis et al., 1991, 1995a; Ohzawa et al., 1996) for individual simple cells, that there can be large differences in shape (spatial phase) between RF profiles measured separately through the left and right eyes. Such differences are particularly prevalent among neurons tuned to oblique or vertical orientations, but not among those tuned to near-horizontal orientations (DeAngelis et al., 1991).

During the course of this study, we had occasion to map RFs through both eyes for three pairs of nearby simple cells. Figure 17
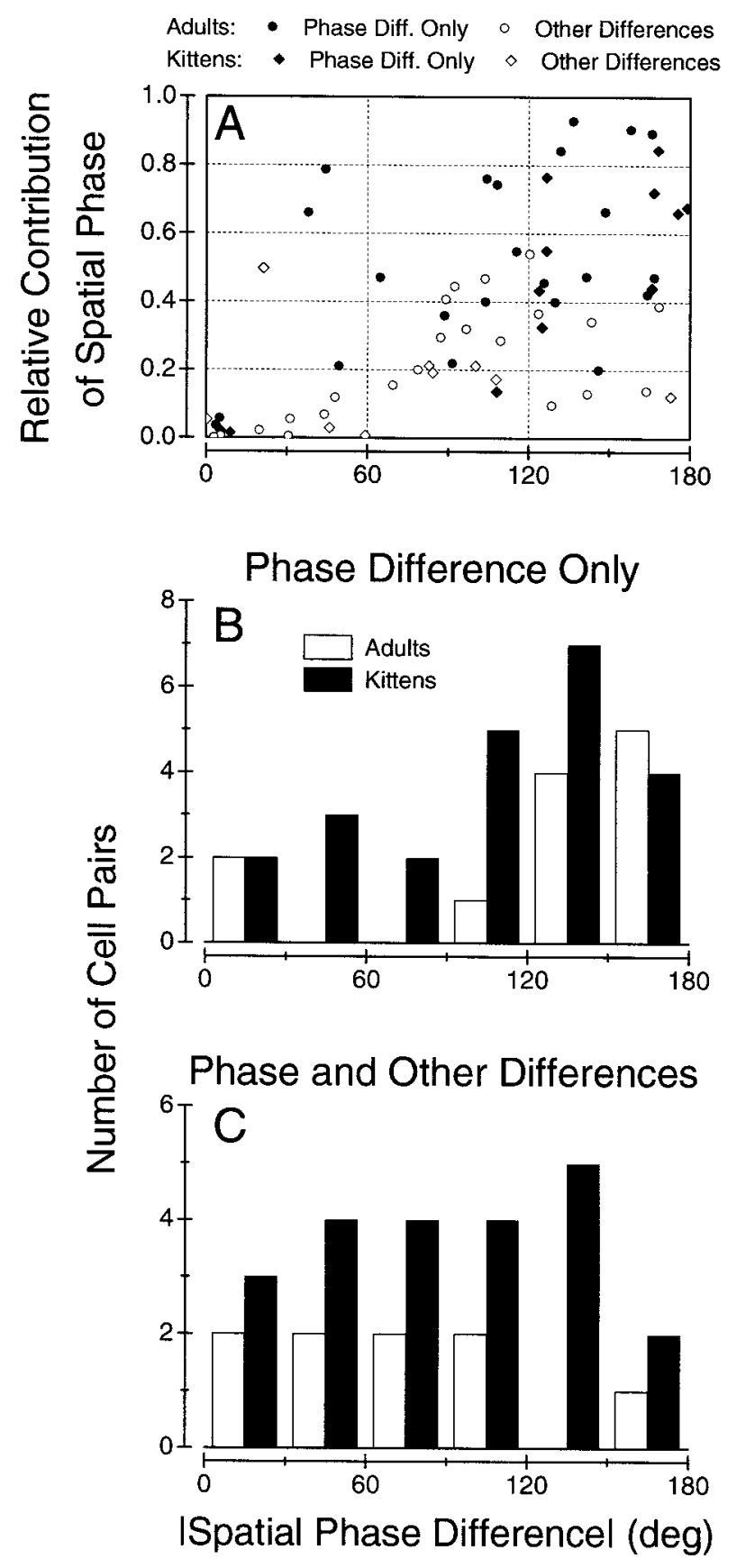

Figure 16. Analysis of spatial phase differences between pairs of simple cells. $A$, The relative contribution of spatial phase to the overall difference in structure between pairs of RFs is plotted as a function of the spatial phase difference between each pair of neurons. Relative contribution of phase is defined as the percent error elevation caused by spatial phase divided by the total percent error elevation caused by all parameters. If the percent error elevation caused by spatial phase is large, and that caused by all other parameters is small (Fig. 14B), then the relative contribution of spatial phase will approach 1.0. Circles and diamonds denote data from adult cats and kittens, respectively. Filled symbols correspond to pairs of neurons for which the relative contribution of all RF parameters, other than spatial phase, does not exceed 0.2. The remaining pairs of neurons are denoted by open symbols. $B$, Histogram showing the distribution of spatial phase differences for pairs of simple cells that differ primarily in terms of spatial phase. Cell pairs included in this distribution are those represented by filled symbols in panel $A$. C, Distribution of spatial phase differences for pairs of simple cells that have a substantial relative contribution from parameters other than spatial phase (these data correspond to the open symbols in panel $A$ ). 

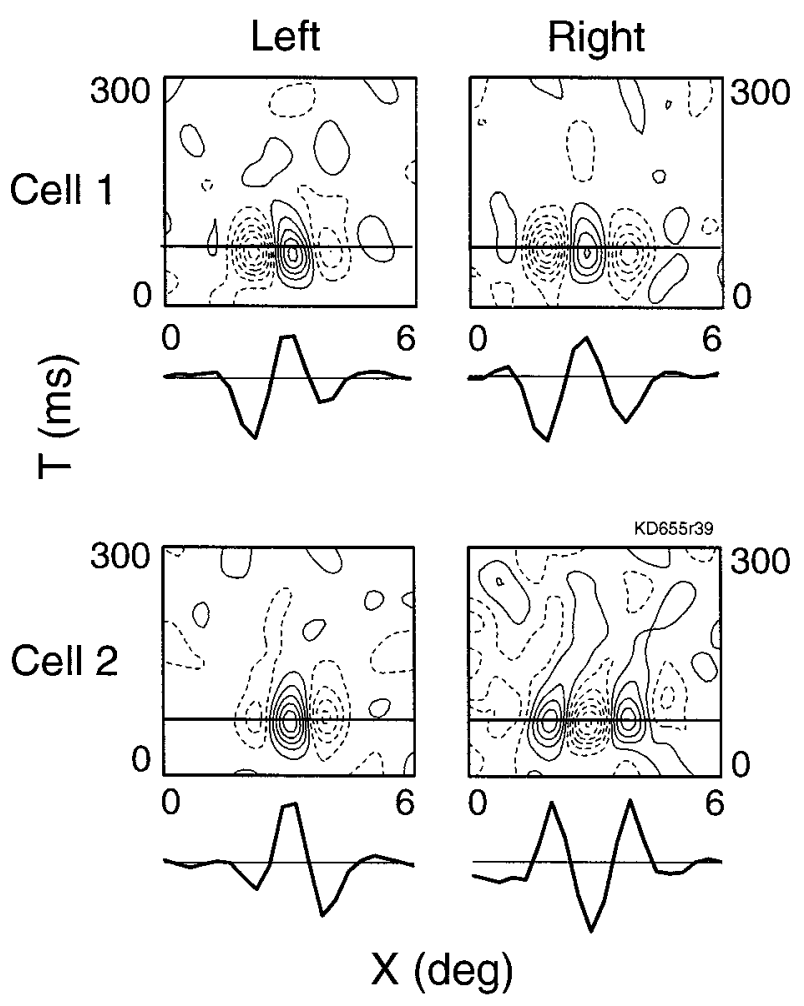

Figure 17. Receptive field profiles for a pair of binocular simple cells (KD655R39). Top row, $X-T$ profiles and $X$ cross sections, as measured through both the left eye and the right eye. This cell has an interocular phase difference of $45^{\circ}$ (Table 1 ) and a preferred orientation that is $27^{\circ}$ from vertical. Bottom row, RF profiles measured through the left and right eyes for a second simple cell, recorded simultaneously. This neuron has an interocular phase difference of $126^{\circ}$ and a preferred orientation that is $18^{\circ}$ from vertical.

shows RF profiles for one such pair. Measured through the left eye, this pair of neurons has a spatial phase difference of $120^{\circ}$ (Fig. 17, left panels). There is also a positional shift, however, such that the two neurons have a central, overlapping bright-excitatory subregion. This results in a positive similarity index, $\mathrm{SI}_{X-T}^{\mathrm{L}}=$ 0.63 . For the right eye, there is a spatial phase difference of $159^{\circ}$ (near antiphase) between the two neurons, which results in a negative similarity index, $\mathrm{SI}^{\mathrm{R}}{ }_{X-T}=-0.59$. By analogy to Equation 1 , we compute a similarity index across both eyes as follows:

$$
\begin{aligned}
& \mathrm{SI}_{X-T}^{B} \\
& =\frac{\sum_{X-T}\left(U^{\mathrm{L}}(X, T) * V^{\mathrm{L}}(X, T)\right)+\sum_{X-T}\left(U^{\mathrm{R}}(X, T) * V^{\mathrm{R}}(X, T)\right)}{\sqrt{\sum_{X-T} U^{\mathrm{L}}(X, T)^{2} \sum_{X-T} V^{\mathrm{L}}(X, T)^{2}}+\sqrt{\sum_{X-T} U^{\mathrm{R}}(X, T)^{2} \sum_{X-T} V^{\mathrm{R}}(X, T)^{2}}}
\end{aligned}
$$

where $\mathrm{U}^{\mathrm{L}}(X, T)$ and $\mathrm{V}^{\mathrm{L}}(X, T)$ are $\mathrm{RF}$ profiles of the two cells as measured through the left eye, and $\mathrm{U}^{\mathrm{R}}(X, T)$ and $\mathrm{V}^{\mathrm{R}}(X, T)$ are RF profiles for the right eye. For the cell pair of Figure 17, we obtain a binocular SI value close to zero, $\mathrm{SI}^{\mathrm{B}}{ }_{X-T}=0.05$. Thus, the binocular RFs of these two neurons are essentially uncorrelated.

Table 1 summarizes the data for this pair of cells (KD655R39), as well as two other binocular pairs. Interestingly, in all three cases, $\mathrm{SI}_{X-T}^{\mathrm{L}}$ and $\mathrm{SI}^{\mathrm{R}}{ }_{X-T}$ have opposite sign, resulting in a binocular index, $\mathrm{SI}^{\mathrm{B}}{ }_{X-T}$, which is much closer to zero than either of the monocular indices. Although this is a small sample of neurons, the data suggest that RFs of nearby simple cells may generally be quite different when one considers all relevant response dimensions.

\section{DISCUSSION}

In this study, we examined the micro-organization of columns within striate cortex by obtaining spatiotemporal RF profiles for pairs of neighboring simple cells. Our results provide the following new insights: (1) when their full $X-Y-T$ profiles are considered, the RFs of nearby simple cells are rarely very similar. The value of $\mathrm{SI}_{X-Y-T}$ seldom exceeds 0.5 (Fig. 3). Moreover, if we take into account a fourth response dimension, binocular disparity, then the similarity of nearby RFs will likely be reduced even further (Table 1). Because the strength of correlated discharge between neighboring cells increases with the degree of similarity between the RFs (Fig. 6), cells with highly similar spatiotemporal RFs tend to carry redundant information. Thus, the local diversity of spatial phase and the general dissimilarity between RFs of nearby neurons may allow for noise reduction through response pooling (discussed below). (2) Several different response variables are significantly clustered within striate cortex. However, the strength of clustering varies markedly from one variable to the next. As established previously, preferred orientation and spatial frequency are strongly clustered. In addition, RF size, response latency, response duration, and preferred temporal frequency exhibit significant clustering. Thus, the functional architecture of primary visual cortex is more complex than is commonly assumed and modeled. (3) RF shape (spatial phase) is locally diverse and exhibits no clustering. Within a local region of striate cortex, where preferred orientation and spatial frequency are approximately constant, spatial phase varies widely from cell to cell. This local diversity of phase may have important implications for the construction of complex cell RFs (discussed below).

\section{Clustering and topography}

Neurons in striate cortex respond selectively to many different parameters of a visual stimulus, and our results show that nearby neurons have similar preferences for many of these parameters. In addition, at least some of these parameters (e.g., receptive field location and orientation preference) are mapped topographically, meaning that they vary smoothly over portions of the cortical surface. However, to organize neurons within the cortical sheet according to multiple parameters, smoothness must be broken. Thus, for example, there are discontinuities in the maps of orientation preference (e.g., Bonhoeffer and Grinvald, 1991) and receptive field position (e.g., Das and Gilbert, 1997).

In dimension-reducing models, each response parameter is an axis in an N-dimensional space (Durbin and Mitchison, 1990; Swindale, 1992, 1996). The cortex is then a two-dimensional sheet that spans the $\mathrm{N}$-dimensional space in as smooth a manner as possible. Only if laminar variations are allowed, which have been suggested for spatial frequency (Maffei and Fiorentini, 1977), can the cortex be considered a three-dimensional volume. Our clustering analysis highlights some of the limitations of these types of models. First, we show that simple cells are clustered along several stimulus dimensions. Although we cannot state whether our clusters reflect columnar organization or laminar organization, we can assert that as many as eight dimensions (the six reported here, ocular dominance, and retinotopy) are clustered within a three-dimensional piece of cortex. It remains to be seen how the predictions of dimension-reducing models will change 
Table 1. Summary of spatial phase and similarity index data for three pairs of binocular simple cells

\begin{tabular}{|c|c|c|c|c|c|c|c|}
\hline \multirow[b]{2}{*}{ Cell pair } & \multicolumn{3}{|l|}{ Left } & \multicolumn{3}{|l|}{ Right } & \multirow[b]{2}{*}{ Binoc. $\mathrm{SI}^{\mathrm{BX}-\mathrm{T}}$} \\
\hline & $\mathrm{P}_{1}$ & $\mathrm{P}_{2}$ & $\mathrm{SI}^{\mathrm{LX}-\mathrm{T}}$ & $\mathrm{P}_{1}$ & $\mathrm{P}_{2}$ & $\mathrm{SI}^{\mathrm{R}_{\mathrm{T}-\mathrm{T}}}$ & \\
\hline KD655R39 & $-80.6^{\circ}$ & $39.8^{\circ}$ & 0.63 & $-35.5^{\circ}$ & $-193.8^{\circ}$ & -0.59 & 0.05 \\
\hline KD021R10 & $-67.1^{\circ}$ & $-172.0^{\circ}$ & -0.54 & $-61.7^{\circ}$ & $-65.4^{\circ}$ & 0.70 & 0.12 \\
\hline BK318R25 & $297.5^{\circ}$ & $131.3^{\circ}$ & -0.85 & $249.9^{\circ}$ & $141.6^{\circ}$ & 0.23 & -0.03 \\
\hline
\end{tabular}

P1, P2, Spatial phase of cell 1, cell 2; $\mathrm{SI}^{\mathrm{L}_{\mathrm{x}-\mathrm{t}}}, \mathrm{SI}^{\mathrm{R}_{\mathrm{x}-\mathrm{t}}}$, monocular similarity indices for the left and right eyes; $\mathrm{SI}^{\mathrm{B}_{\mathrm{X}-\mathrm{t}}}$, similarity index for both eyes.

when these additional parameters of functional organization are incorporated. Second, we show that all parameters in the $\mathrm{N}$-dimensional space are not equal in terms of the strength of clustering (see Fig. 12). Perhaps this is a necessary consequence of mapping several variables onto the cortical sheet. Thus, it would be interesting to see, for example, whether the incorporation of these new constraints into dimension-reducing models would lead to patterns of functional organization more consistent with recent experimental data (Das and Gilbert, 1997) concerning retinotopy and orientation.

\section{Correlated discharge and RF similarity}

The primary quantitative analyses that have been applied to pairs of neurons are cross-correlation of spike trains and a covariance measure of spike counts. Covariance measures examine how the spike counts of two cells covary with repeated presentation of the same stimulus and have been obtained from multiple visual areas, including area 17 of the cat (van Kan et al., 1985), area MT of the macaque (Zohary et al., 1994), and inferotemporal cortex of the macaque (Gawne and Richmond, 1993). Interestingly, in all of these areas, the covariance values are similar, $\sim 0.15-0.2$. Although these values are also similar to our average threedimensional similarity value $\left(\mathrm{SI}_{X-Y-T}\right)$, it is important to realize that these are fundamentally different metrics. Covariance measures the shared noise in the responses of two cells; similarity measures the overlap between receptive fields, which is more akin to shared signal rather than shared noise. One can imagine two cells that have zero similarity but a high covariance simply because they share a common noise source. Alternatively, one can imagine a pair of cells with identical receptive fields that do not share a common noise source and therefore display no covariance. The quantitative agreement of our average $\mathrm{SI}_{X-Y-T}$ and average covariance measures may therefore be coincidental. However, this does not necessarily mean that the two metrics are independent. van Kan et al. (1985) reported that high covariance was observed only between cells with similar receptive field properties. Because similarity was not quantified in that study, and covariance was not examined in our study, the exact nature of the relationship remains an open question.

\section{Spatial phase distribution and hierarchical organization of striate cortex}

A striking result of our study is that no clustering was found for the spatial phase of nearby RFs (Fig. 11G, 12). This finding lies in stark contrast to results from computational studies of cortical development that predict the emergence of spatial phase columns in striate cortex (Miller, 1992; Miyashita and Tanaka, 1992). Although our results are unequivocal on this point, there may be overriding reasons for the lack of clustering of spatial phase.

One likely possibility is that a variety of spatial phases is needed to efficiently construct complex cell RFs from the outputs of simple cells. Modeling studies indicate that a complex cell with reasonable physiological properties can be constructed from a group of four or more simple cells having different spatial phases (Pollen et al., 1989; Ohzawa et al., 1990; Emerson et al., 1992; Qian, 1994). If simple cells with different spatial phases are located close to one another and to the target complex cell, then this connectivity can be implemented with a minimum total length of dendrites and axons. On the other hand, if simple cells are organized in phase columns, then the connections to a target complex cell would have to extend across several different columns. Thus, a columnar organization for spatial phase may be too costly in terms of connections. In addition, the local diversity of simple cell phases may be beneficial from the standpoint of response pooling (discussed below).

Our results loosely agree with previous work on the spatial phase relationships between pairs of nearby simple cells. Pollen and colleagues (Pollen and Ronner, 1981; Liu et al., 1992) reported that nearby simple cells tend to occur in either quadrature phase $\left(90^{\circ}\right.$ difference) or antiphase $\left(180^{\circ}\right.$ difference). Our data from pairs of simple cells that differ only in phase also reveal a preponderance of phase differences in the range from 90 to $180^{\circ}$. However, our distribution of phase differences is not bimodal (with peaks near 90 and $180^{\circ}$ ), suggesting that quadrature and antiphase pairs are not discrete classes.

\section{Development}

We examined whether the functional micro-organization of striate cortex matures noticeably after 4 postnatal weeks. Four-weekold animals were chosen for study because the cortex is highly malleable at this age. Monocular deprivation, for example, is known to cause reorganization of ocular dominance columns, with a critical period of susceptibility that peaks at $\sim 4$ postnatal weeks (Olson and Freeman, 1980). However, this susceptibility to modification does not necessarily imply that normal animals at this age have immature functional organization. Single-unit electrophysiology suggests that the spatial properties of striate receptive fields are quite mature by 4 postnatal weeks (Braastad and Heggelund, 1985; DeAngelis et al., 1993a) (see also, Fig. $11 A, C, E)$. In contrast, temporal RF properties are still quite immature at 4 weeks of age (DeAngelis et al., 1993a) (see also, Fig. $11 B, D, F)$.

Our present results suggest that most aspects of the local organization of striate cortex are mature by 4 postnatal weeks. The overall pattern of similarity between RFs of nearby neurons is indistinguishable between cats and kittens (Figs. 3, 4). Moreover, in both age groups, there is a clear increase in correlated discharge between neighboring neurons as a function of RF similarity (Fig. 6). The strength of local clustering for different $\mathrm{RF}$ attributes is also quite similar between the two age groups (Fig. 12). In addition, spatial phase is the largest source of difference between nearby RFs for both cats and kittens (Fig. 
$15 A$ ). Thus, in many respects, the functional micro-organization of cat striate cortex appears to be mature by 4 postnatal weeks.

\section{Clustering and response pooling}

Our analyses reveal that different receptive field parameters are clustered to varying degrees within striate cortex. These differences raise interesting questions: why do some parameters form bases for cortical organization, whereas others do not? Why is clustering for orientation stronger than for spatial frequency, and why is there no tendency for spatial phase to cluster?

One interesting possibility is that these patterns of clustering reflect the need to pool responses across populations of nearby neurons. The benefits of pooling are well documented: because neuronal responses are noisy, combining the responses of several similarly tuned neurons can lead to improved signal fidelity (for review, see Parker and Newsome, 1998). Pooling can potentially reduce noise that originates within the nervous system as well as noise that originates from external influences. Pooling across neurons also reduces the need for temporal integration, allowing a reliable signal to be extracted from a brief epoch of neuronal discharge.

Consider, for example, the problem of extracting a reliable orientation signal from a group of simple cells. Because simple cell responses depend on the exact position of image features within the RF, positional jitter in the image (e.g., from microsaccades) contributes noise to the orientation signal produced by a single unit. For a pair of nearby simple cells with RFs in quadrature phase, however, responses to positional variations will be uncorrelated. Thus, if we care about orientation and do not care about luminance variations smaller than the RF, pooling across this pair of neurons will improve the orientation signal substantially. In reality, this improvement in signal-to-noise ratio is limited because the majority of cell pairs do show some correlation in their responses. Zohary et al. (1994) show that even small amounts of correlation limit the benefits of pooling to pool sizes of 50-100 neurons.

Our data suggest that pooling across simple cells with different spatial phases may be a viable way to improve signal quality. Because only neurons with similar RFs tend to have correlated discharges (Fig. 6), there may be considerable advantage to pooling across neurons with dissimilar spatial phases. Such pooling may be substantially facilitated by having spatial phase be locally diverse within striate cortex while other parameters (e.g., orientation) are tightly clustered; pooling across phase would simply involve combining the responses of several nearby simple cells without a need for selective local connections. In this regard, the clustering indices of Figure 6 may give us an indication of which parameters are the most likely to be "improved" by pooling: orientation and spatial frequency.

Fundamentally, the advantage of this form of pooling relies on some response parameters being clustered within a given visual area, whereas others are not. Given that neurons in most cortical areas exhibit tuning along multiple stimulus dimensions, the idea of using local variations in one parameter to maximize pooling effects for other parameters might be a general strategy of information processing in the cortex.

\section{REFERENCES}

Adelson EH, Bergen JR (1985) Spatiotemporal energy models for the perception of motion. J Opt Soc Am A 2:284-299.

Albrecht DG, Geisler WS (1991) Motion selectivity and the contrastresponse function of simple cells in the visual cortex. Vis Neurosci 7:531-546.
Bartfeld E, Grinvald A (1992) Relationships between orientationpreference pinwheels, cytochrome oxidase blobs, and oculardominance columns in primate striate cortex. Proc Natl Acad Sci USA 89:11905-11909.

Berardi N, Bisti S, Cattaneo A, Fiorentini A, Maffei L (1982) Correlation between the preferred orientation and spatial frequency of neurones in visual areas 17 and 18 of the cat. J Physiol (Lond) 323:603-618.

Berman NE, Wilkes ME, Payne BR (1987) Organization of orientation and direction selectivity in areas 17 and 18 of cat cerebral cortex. J Neurophysiol 58:676-699.

Blasdel GG (1992) Orientation selectivity, preference, and continuity in monkey striate cortex. J Neurosci 12:3139-3161.

Bonhoeffer T, Grinvald A (1991) Iso-orientation domains in cat visual cortex are arranged in pinwheel-like patterns. Nature 353:429-431.

Bonhoeffer T, Kim DS, Malonek D, Shoham D, Grinvald A (1995) Optical imaging of the layout of functional domains in area 17 and across the area $17 / 18$ border in cat visual cortex. Eur J Neurosci 7:1973-1988.

Braastad BO, Heggelund P (1985) Development of spatial receptivefield organization and orientation selectivity in kitten striate cortex. J Neurophysiol 53:1158-1178.

Das A, Gilbert CD (1997) Distortions of visuotopic map match orientation singularities in primary visual cortex. Nature 387:594-598.

DeAngelis GC, Ohzawa I, Freeman RD (1991) Depth is encoded in the visual cortex by a specialized receptive field structure. Nature 352:156-159.

DeAngelis GC, Ohzawa I, Freeman RD (1993a) Spatiotemporal organization of simple-cell receptive fields in the cat's striate cortex. I. General characteristics and postnatal development. J Neurophysiol 69:1091-1117.

DeAngelis GC, Ohzawa I, Freeman RD (1993b) Spatiotemporal organization of simple-cell receptive fields in the cat's striate cortex. II. Linearity of temporal and spatial summation. J Neurophysiol 69:1118-1135.

DeAngelis GC, Ohzawa I, Freeman RD (1995a) Neuronal mechanisms underlying stereopsis: how do simple cells in the visual cortex encode binocular disparity? Perception 24:3-31.

DeAngelis GC, Ohzawa I, Freeman RD (1995b) Receptive-field dynamics in the central visual pathways. Trends Neurosci 18:451-458.

DeBoer E, Kuyper P (1968) Triggered correlation. IEEE Trans Biomed Eng 15:169-179.

Durbin R, Mitchison G (1990) A dimension reduction framework for understanding cortical maps. Nature 343:644-647.

Emerson RC, Bergen JR, Adelson EH (1992) Directionally selective complex cells and the computation of motion energy in cat visual cortex. Vision Res 32:203-218.

Field DJ, Tolhurst DJ (1986) The structure and symmetry of simple-cell receptive-field profiles in the cat's visual cortex. Proc R Soc Lond B Biol Sci 228:379-400.

Freeman RD, Ohzawa I (1992) Development of binocular vision in the kitten's striate cortex. J Neurosci 12:4721-4736.

Gaska JP, Jacobson LD, Chen HW, Pollen DA (1994) Space-time spectra of complex cell filters in the macaque monkey: a comparison of results obtained with pseudowhite noise and grating stimuli. Vis Neurosci 11:805-821.

Gawne TJ, Richmond BJ (1993) How independent are the messages carried by adjacent inferior temporal cortical neurons? J Neurosci 13:2758-2771.

Ghose GM, Freeman RD, Ohzawa I (1994a) Local intracortical connections in the cats visual cortex: postnatal development and plasticity. J Neurophysiol 72:1290-1303.

Ghose GM, Ohzawa I, Freeman RD (1994b) Receptive-field maps of correlated discharge between pairs of neurons in the cats visual cortex. J Neurophysiol 71:330-346.

Ghose GM, Ohzawa I, Freeman RD (1995) A flexible pc-based physiological monitor for animal experiments. J Neurosci Methods 62:7-13.

Hubel DH, Wiesel TN (1974) Sequence regularity and geometry of orientation columns in the monkey striate cortex. J Comp Neurol 158:267-293.

Hubel DH, Wiesel TN (1977) Ferrier lecture. Functional architecture of macaque monkey visual cortex. Proc R Soc Lond B Biol Sci 198:1-59.

Hubel DH, Wiesel TN, Stryker MP (1977) Orientation columns in macaque monkey visual cortex demonstrated by the 2-deoxyglucose autoradiographic technique. Nature 269:328-330.

Hübener M, Shoham D, Grinvald A, Bonhoeffer T (1997) Spatial rela- 
tionships among three columnar systems in cat area 17. J Neurosci 17:9270-9284.

Jones JP, Palmer LA (1987) The two-dimensional spatial structure of simple receptive fields in cat striate cortex. J Neurophysiol 58:1187-1211.

Liu Z, Gaska JP, Jacobson LD, Pollen DA (1992) Interneuronal interaction between members of quadrature phase and anti-phase pairs in the cat's visual cortex. Vision Res 32:1193-1198.

Maffei L, Fiorentini A (1977) Spatial frequency rows in the striate visual cortex. Vision Res 17:257-264.

McLean J, Palmer LA (1989) Contribution of linear spatiotemporal receptive field structure to velocity selectivity of simple cells in area 17 of cat. Vision Res 29:675-679.

McLean J, Raab S, Palmer LA (1994) Contribution of linear mechanisms to the specification of local motion by simple cells in areas 17 and 18 of the cat. Vis Neurosci 11:271-294.

Melssen WJ, Epping WJ (1987) Detection and estimation of neural connectivity based on cross-correlation analysis. Biol Cybern $57: 403-414$.

Miller KD (1992) Development of orientation columns via competition between ON- and OFF-center inputs. NeuroReport 3:73-76.

Miyashita M, Tanaka S (1992) A mathematical model for the selforganization of orientation columns in visual cortex. NeuroReport 3:69-72.

Mountcastle VB (1997) The columnar organization of the neocortex. Brain 120:701-722.

Movshon JA, Thompson ID, Tolhurst DJ (1978) Spatial summation in the receptive fields of simple cells in the cat's striate cortex. J Physiol (Lond) 283:53-77.

Obermayer K, Blasdel GG (1993) Geometry of orientation and ocular dominance columns in monkey striate cortex. J Neurosci 13:4114-4129.

Ohzawa I, DeAngelis GC, Freeman RD (1990) Stereoscopic depth discrimination in the visual cortex: neurons ideally suited as disparity detectors. Science 249:1037-1041.

Ohzawa I, DeAngelis GC, Freeman RD (1996) Encoding of binocular disparity by simple cells in the cat's visual cortex. J Neurophysiol 75:1779-1805.

Olson CR, Freeman RD (1980) Profile of the sensitive period for monocular deprivation in kittens. Exp Brain Res 39:17-21.

Parker AJ, Newsome WT (1998) Sense and the single neuron: probing the physiology of perception. Annu Rev Neurosci 21:227-277.

Payne BR, Berman N (1983) Functional organization of neurons in cat striate cortex: variations in preferred orientation and orientation selectivity with receptive-field type, ocular dominance, and location in visual-field map. J Neurophysiol 49:1051-1072.

Payne BR, Berman N, Murphy EH (1980) Organization of direction preferences in cat visual cortex. Brain Res 211:445-450.

Pollen DA, Ronner SF (1981) Phase relationships between adjacent simple cells in the visual cortex. Science 212:1409-1411.
Pollen DA, Gaska JP, Jacobson LD (1989) Physiological constraints on models of visual cortical function. In: Models of brain function (Cotterill RMJ, ed), pp 115-135. Cambridge, UK: Cambridge UP.

Qian N (1994) Computing stereo disparity and motion with known binocular cell properties. Neural Comput 6:390-404.

Reid RC, Alonso JM (1995) Specificity of monosynaptic connections from thalamus to visual cortex. Nature 378:281-284.

Reid RC, Soodak RE, Shapley RM (1991) Directional selectivity and spatiotemporal structure of receptive fields of simple cells in cat striate cortex. J Neurophysiol 66:505-529.

Shmuel A, Grinvald A (1996) Functional organization for direction of motion and its relationship to orientation maps in cat area 18. J Neurosci 16:6945-6964.

Shoham D, Hübener M, Schulze S, Grinvald A, Bonhoeffer T (1997) Spatio-temporal frequency domains and their relation to cytochrome oxidase staining in cat visual cortex. Nature 385:529-533.

Swindale NV (1992) Elastic nets, travelling salesmen, and cortical maps. Curr Biol 2:429-431.

Swindale NV (1996) Visual cortex: looking into a Klein bottle. Curr Biol 6:776-779.

Tolhurst DJ, Dean AF, Thompson ID (1981) Preferred direction of movement as an element in the organization of cat visual cortex. Exp Brain Res 44:340-342.

Tolhurst DJ, Thompson ID (1982) Organization of neurones preferring similar spatial frequencies in cat striate cortex. Exp Brain Res 48:217-227

Tootell RB, Silverman MS, De Valois RL (1981) Spatial frequency columns in primary visual cortex. Science 214:813-815.

Ts'o DY, Gilbert CD, Wiesel TN (1986) Relationships between horizontal interactions and functional architecture in cat striate cortex as revealed by cross-correlation analysis. J Neurosci 6:1160-1170.

Ts'o DY, Frostig RD, Lieke EE, Grinvald A (1990) Functional organization of primate visual cortex revealed by high resolution optical imaging. Science 249:417-420.

van Kan PL, Scobey RP, Gabor AJ (1985) Response covariance in cat visual cortex. Exp Brain Res 60:559-563.

Watson AB, Ahumada Jr A (1985) Model of human visual-motion sensing. J Opt Soc Am A 2:322-341.

Weliky M, Bosking WH, Fitzpatrick D (1996) A systematic map of direction preference in primary visual cortex. Nature 379:725-728.

Wilson JR, Sherman SM (1976) Receptive-field characteristics of neurons in cat striate cortex: changes with visual field eccentricity. J Neurophysiol 39:512-533.

Zohary E, Shadlen MN, Newsome WT (1994) Correlated neuronal discharge rate and its implications for psychophysical performance. Nature 370:140-143. 